\title{
GLOBAL ANALYSIS ON PL-MANIFOLDS ${ }^{1}$ \\ BY \\ NICOLAE TELEMAN
}

\begin{abstract}
The paper deals mainly with combinatorial structures; in some cases we need refinements of combinatorial structures. Riemannian metrics are defined on any combinatorial manifold $M$.

The existence of distance functions and of Riemannian metrics with "constant volume density" implies smoothing.

A geometric realization of $\mathrm{PL}(m) / O(m)$ is given in terms of Riemannian metrics.

A graded differential complex $\Omega^{*}(M)$ is constructed: it appears as a subcomplex of Sullivan's complex of piecewise differentiable forms. In the complex $\Omega^{*}(M)$ the operators $d, *, \delta, \Delta$ are defined.

A Rellich chain of Sobolev spaces is presented. We obtain a Hodge-type decomposition theorem, and the Hodge homomorphism is defined and studied. We study also the combinatorial analogue of the signature operator.
\end{abstract}

\section{INTRODUCTION}

This paper is a slightly modified version of my $\mathrm{Ph} . \mathrm{D}$. thesis at the Massachusetts Institute of Technology.

The departure point of this study was a problem proposed by I. M. Singer [S], that is the problem of the geometrical realization of Sullivan's orientation class $\left[\mathrm{Su}_{2}\right]$. This study has to be considered as a first approach towards the solution of this problem.

In this paper we extend, by analogy, some techniques of partial differential equations involved in the solution of the signature theorem, from the category of smooth manifolds to that of combinatorial manifolds. To our knowledge, this paper constitutes the first tentative step of this nature.

We show that some phenomena in the smooth case persist even in the combinatorial case, while others do not.

This paper is divided into three chapters.

After the first two preparatory sections of Chapter I, we define in $\$ 3$ a graded differential complex $\Omega^{*}(M)$ which is a subcomplex of Sullivan's complex $A^{*}(M)$ of piecewise differentiable forms. In the complex $\Omega^{*}(M)$ the

Received by the editors June 21, 1977 and, in revised form, June 22, 1978.

AMS (MOS) subject classifications (1970). Primary 57D10, 58-xx, 58G05; Secondary 57C99, $58 \mathrm{G} 10$.

Key words and phrases. Riemannian structure on PL-manifold, constant volume density, distance function, distributions, Sobolev spaces, Hodge theory, signature operator.

${ }^{1}$ Work partially supported by a Teaching Assistantship at the Massachusetts Institute of Technology.

() 1979 American Mathematical Society 
star operator * is defined, and hence it makes sense to define the exterior co-derivative $\delta$ and the Laplace-Beltrami operator $\Delta$ (\$4). In our study, the elements of the complex $\Omega^{*}(M)$ play the role of test functions.

In Chapter II we study some smoothing problems. We show in $\$ 1$ (Theorem 1.2) that if a combinatorial manifold admits a Riemannian metric with the property that, for any point $x$ in it, the sum of measures of all solid angles tangent to the simplices of the manifold which contain $x$ is the measure of the solid angle around a point in Euclidean space, then the Riemannian metric defines a smoothing of the manifold; in particular, $M$ is smoothable.

The local measure of a Riemannian metric seems to us to play a central role in the analysis of combinatorial manifolds. Theorem 1.2 suggests also that it does not make sense to look for special Riemannian metrics on PL-manifolds.

Another consequence of Theorem 1.2 is Theorem 2.1 of $\$ 2$ which asserts that if a combinatorial manifold admits a distance function of class $C^{3}$, then $M$ is smoothable, showing so that Bidal-de Rham method for constructing a parametrix for the Laplacian is no longer applicable on PL-manifolds.

In $\$ 3$ we obtain as an application of Theorem 1.2 some fundamental results from the theory of smoothing.

Chapter III presents a combinatorial Hodge theory and studies a combinatorial analogue of the signature operator. In $\$ 1$ we define distributions in PL-manifolds. In $\$ 2$ we introduce natural norms on $\Omega^{*}(M)$ and we define Sobolev spaces $H_{s}^{P}(M)$ on PL-manifolds. At the end of $\$ 2$ we show that the sequence of Sobolev spaces $\left\{H_{s}^{p}\right\}_{s}, 0<p<\operatorname{dim} M$, is a Rellich chain. In $\S 3$ we show that the Sobolev spaces $H_{s}^{p}$ are spaces of distributions. In $\$ 4$ we compute homology of the complex of distributional forms (currents) on $M$ : we show this cohomology is $H^{*}(M, R)$, thus obtaining a complete analogue of de Rham's theorem.

In $\$ 5$ we obtain a Hodge-type decomposition theorem (Theorem 5.1). Theorem 5.2 shows that the Hodge homomorphism (which we define accordingly) is a monomorphism on the space of the harmonic forms which stay in the Sobolev space $H_{1}^{*}$, while the Hodge homomorphism is an epimorphism on the space of the harmonics which stay in $H_{-1}^{*}$. In $\$ 6$ we study the combinatorial analogue of the signature operator and we show (Theorem 6.3) that the defect $\theta$ depends only on the cokernel of the Hodge homomorphism in the middle dimension.

Acknowledgements. I wish to thank Professor I. M. Singer who supervised my research at M.I.T. I express my gratitude for his continuous interest in my research, for the useful conversations and for his encouragement. I also wish to thank Professor J. Munkres for many useful conversations and for his remarks about my results and to acknowledge stimulating discussions with Professor D. G. Schaeffer. I thank the staff of the Mathematics Department 
which granted me a Teaching Assistantship during my residence at M.I.T. I thank the referee for valuable observations which led to improvement of the text.

\section{Chapter I. Preliminaries}

1. Preliminaries. Throughout this paper $M^{m}$ denotes a compact oriented combinatorial manifold of dimension $m$ without boundary.

All simplices of the decomposition of $M^{m}$ are considered closed. Two maximal simplices are said to be "neighboring" if their intersection is a simplex of dimension $m-1$.

2. Riemannian structures. A Riemannian structure of class $C^{k}, 0<k<\infty$, on $M$ is, by definition, a function $\Gamma$ which associates with any maximal simplex $\sigma \in M$ a Riemannian structure $\Gamma_{\sigma}$ of class $C^{k}$ on the (closed) simplex $\sigma$; these Riemannian structures are required to be " $C^{k}$-compatible", a notion which is defined below.

First suppose that $k \geqslant 1$. If $N$ is any smooth manifold (with or without boundary), let $T_{x} N$ denote its tangent space at $x \in N$. If $\sigma$ is a maximal simplex in $M$, if $x$ is an interior point of a $k$-dimensional face $\pi \subset \sigma$ and if $U$ is an open neighborhood of $x$ in $\sigma$, a coordinate chart

$$
\rho: U \rightarrow \mathbf{R}^{k} \times \mathbf{R}^{m-k}
$$

is called "adapted at the boundary" iff:

(i) $\rho(U \cap \pi) \subset \mathbf{R}^{k} \times\{0\}$,

(ii) $\lambda:[0, \varepsilon) \rightarrow U$ being an arc of geodesic, $\lambda(0) \in \pi,\|\dot{\lambda}(0)\|=1, \lambda$ normal at $\pi$, then

$$
\rho(\lambda(t))=\left(0, \ldots, 0, a_{1} t, \ldots, a_{m-k} t\right)+\rho(\lambda(0)),
$$

where $\Sigma_{i} a_{i}^{2}=1$.

Suppose $\sigma, \tau$ are two neighboring simplices in $M$ and $x \in$ Int $\sigma \cap \tau$. Let $\Psi$ be any coordinate system on a small neighborhood $V$ of $x$ in $\sigma \cap \tau$; then there exist only two coordinate systems $\rho$ on a sufficiently small neighborhood $U$ of $x$ in $M$, with $U \cap(\sigma \cap \tau)=V$, such that

(i) $\rho|U \cap \sigma, \rho| U \cap \tau$ are coordinate systems adapted at the boundary,

(ii) $\rho \mid V=\Psi$,

(iii) $\rho(U)=\Psi(V) \times(-\varepsilon, \varepsilon) \subset \mathbf{R}^{m-1} \times \mathbf{R}, \varepsilon>0$.

Any such $\rho$ is called a "bicollared coordinate system" on $U$.

Suppose that $\sigma$ and $\tau$ are two neighboring simplices. We say that $\Gamma_{\sigma}, \Gamma_{\tau}$ are $C^{k}$-compatible iff for any bicollared coordinate system on a neighborhood $U$ of $x \in$ Int $\sigma \cap \tau$, the Riemannian metric $\left(\left(\rho^{-1}\right) * \Gamma_{\sigma},\left(\rho^{-1}\right) * \Gamma_{\tau}\right)$ on $\rho(U)$ is of class $C^{k}$.

We say that the $\Gamma_{\sigma}$ are $C^{k}$-compatible iff for any neighboring simplices $\sigma, \tau$ in $M, \Gamma_{\sigma}$ and $\Gamma_{\tau}$ are $C^{k}$-compatible. 
REMark 2.1. The definition of $C^{k}$-compatibility does not depend on the particular bicollared coordinate system chosen.

If $k=0$, we say that $\Gamma_{\sigma}, \Gamma_{\tau}$ are $C^{0}$-compatible iff their restrictions at $\sigma \cap \tau$ coincide, i.e.,

$$
\Gamma_{\sigma}\left|\sigma \cap \tau=\Gamma_{\tau}\right| \sigma \cap \tau .
$$

REMARK 2.2. If $\Gamma_{\sigma}, \Gamma_{\tau}$ are $C^{k}$-compatible, they are $C^{l}$-compatible, for any $0<l<k$.

REMARK 2.3. In spite of the fact that the definition of $C^{k}$-compatibility involves relationships only between Riemannian metrics of neighboring simplices, it implies relations between the Riemannian metrics of any two intersecting simplices of $M$. Really, the relations (2.2) make sense and remain valid, by continuity, even for $x$ at the boundary of $\sigma \cap \tau$.

If $\sigma, \tau$ are two intersecting $m$-simplices in $M^{m}$, and $\Gamma$ is a $C^{0}$-Riemannian structure on $M$, then

$$
\Gamma_{\sigma}\left|\sigma \cap \tau=\Gamma_{\tau}\right| \sigma \cap \tau
$$

whatever $\operatorname{dim} \sigma \cap \tau$ is. Indeed, in $\operatorname{Star}(\sigma, M)$ there exists a chain of $m$-simplices $\sigma=\alpha_{0}, \alpha_{1}, \ldots, \alpha_{p}=\tau$, with $\alpha_{i-1}$ and $\alpha_{i}$ neighboring, $1<i<p$; hence

$$
\begin{aligned}
\Gamma_{\sigma}\left|\sigma \cap \alpha_{1}=\Gamma_{\alpha_{1}}\right| \sigma \cap \alpha_{1}, \quad \Gamma_{\alpha_{1}}\left|\alpha_{1} \cap \alpha_{2}=\Gamma_{\alpha_{2}}\right| \alpha_{1} \cap \alpha_{2}, \ldots, \\
\Gamma_{\alpha_{p-1}}\left|\alpha_{p-1} \cap \tau=\Gamma_{\tau}\right| \alpha_{p-1} \cap \tau
\end{aligned}
$$

which proves (2.3).

REMARK 2.4. Any combinatorial manifold $M$ admits a Riemannian metric $\Gamma$ with all the $\Gamma_{\sigma}$ flat. Indeed, it is enough to embed $M$ linearly in a Euclidean space and take the induced metric on each simplex of the image.

REMARK 2.5. If $\Gamma$ is a Riemannian metric of class $C^{0}$ on $M$ and if all the $\Gamma_{\text {。 }}$ are flat, then $\Gamma$ is of class $C^{\infty}$.

3. Differential forms. Suppose $\Gamma$ is a Riemannian structure of class $C^{\infty}$ on $M$. A real (resp., complex) differential form of degree $r$ on $M$ is, by definition, a function $\omega$ which associates with any maximal simplex $\sigma \in M$ a real (resp., complex) differential form $\omega_{\sigma}$ of degree $r$ on $\sigma$.

The differential form $\omega$ is, by definition, of class $C^{k}, 0<k<\infty$, with respect to $\Gamma$, iff any $\omega_{\sigma}$ is of class $C^{k}$ and for any two neighboring cells $\sigma, \tau$ and for any bicollared coordinate system $\rho$ on $U$, a small neighborhood of any point $x \in \operatorname{Int}(\sigma \cap \tau)$, the form

$$
\left(\left(\rho^{-1}\right) * \omega_{\sigma},\left(\rho^{-1}\right) * \omega_{\tau}\right)
$$

is of class $C^{k}$ on $\rho(U) \subset \mathbf{R}^{m}$.

REMARK 3.1. From (3.1) it follows that the restrictions of $\omega_{\sigma}, \omega_{\tau}$ to $\sigma \cap \tau$ are equal:

$$
\omega_{\sigma}\left|\sigma \cap \tau=\omega_{\tau}\right| \sigma \cap \tau
$$


The argument in Remark 2.3 can still be applied; hence (3.2) remains valid for any $\sigma, \tau$, not necessarily neighboring.

Definition 3.1. If $\mathbf{F}=\mathbf{R}$ or $\mathbf{C}$,

$$
\Omega_{\mathbf{F}}^{r}(M)=\left\{\omega \mid \omega \text { is an } F C^{\infty} \text {-form of degree } r \text { on } M\right\} \text {. }
$$

Defintion 3.2. If $\omega \in \Omega^{r}(M)$, we define the differential $d \omega$ of $\omega$ to be the form

$$
(d \omega)_{0}=d\left(\omega_{0}\right), \quad \forall \sigma=m \text {-simplex. }
$$

Definition 3.3. If $\omega$ (resp., $\vartheta$ ) is a form of degree $r$ (resp., $s$ ) on $M$, then $\omega \wedge \vartheta$ is the form of degree $r+s$ :

$$
(\omega \wedge \vartheta)_{\sigma}=\omega_{\sigma} \wedge \vartheta_{\sigma}
$$

Proposition 3.1. For any $\omega \in \Omega(M)$, $\vartheta \in \Omega^{r}(M)$,

(i) $d^{2} \omega=0$,

(ii) $d \omega \in \Omega^{r+1}(M)$,

(iii) $\omega \wedge \vartheta \in \Omega^{r+s}(M)$.

Proof. The proof reduces to the case of $C^{\infty}$ forms on $\mathbf{R}^{m}$.

COROLLARY 3.1. $\Omega_{\mathrm{F}}^{*}(M)=\left\{\Omega_{\mathrm{F}}^{r}(M), d\right\}_{r \in \mathrm{N}}$ is a differential graded algebra.

Definition 3.4. If $\omega \in \Omega^{m}(M)$, we define

$$
\int_{M} \omega=\sum_{\sigma, \operatorname{dim} \sigma=m} \int_{\sigma} \omega_{\sigma}
$$

Proposition 3.2 (Stokes' formula). If $\omega \in \Omega^{m-1}(M)(\partial M=\varnothing)$,

$$
\int_{M} d \omega=0
$$

Proof. We have

$$
\int_{M} d \omega=\sum_{\sigma} \int_{\sigma} d \omega_{\sigma}=\sum_{\sigma} \int_{\partial \sigma} \omega_{\sigma}
$$

If $\alpha$ is any ( $m-1)$-simplex in $M$, and if $\sigma, \tau$ are neighboring simplices with intersection $\alpha$, then (3.2) gives us

$$
\omega_{\sigma}\left|\alpha=\omega_{\tau}\right| \alpha .
$$

The coefficient of $\alpha$ in $\partial \sigma+\partial \tau$ is 0 because the orientations of $\sigma$ and $\tau$ are coherent; hence (3.4).

We recall here the definition of PL-forms over a cell complex (see Sullivan [Su$u_{1}$ ). If $K$ is a cell complex, a PL-form of degree $p$ over $K$ is a function $\omega$ which associates with any cell $\sigma$ of $K$ a $C^{\infty}$ form $\omega$ of degree $p$ over $\sigma$ : these forms $\omega_{0}$ must be compatible in the sense that for any two cells $\sigma, \tau$ of $K$, their restrictions to $\sigma \cap \tau$ must be equal. The set of all PL-forms of degree $p$ 
over $K$ is denoted by $A^{p}(K)$, while $A^{*}(K)=\bigoplus_{p} A^{p}(K)$. If $\omega \in A^{p}(K)$, then $(d \omega)_{\sigma}=d\left(\omega_{\sigma}\right)$ belongs to $A^{p+1}(K)$.

Proposition 3.3. If $M$ is a combinatorial manifold, then $\Omega^{p}(M) \subset A^{p}(M)$.

Proof. It follows from Remark 3.1.

4. Laplace-Beltrami operator. Let $\Gamma$ be a $C^{\infty}$-Riemannian structure on $M$. DefINITION 4.1. If $\omega$ is any $r$-form on $M, *{ }_{r} \omega$ is the $(m-r)$-form:

$$
(*, \omega)_{0}=*{ }_{r}\left(\omega_{\sigma}\right), \quad \sigma=m \text {-simplex, }
$$

the last *, being the usual * -operator defined by $\Gamma_{\sigma}$ (for the definition of * and its properties on smooth Riemannian manifolds see de Rham [RD).

Proposition 4.1. If $\omega \in \Omega^{\prime}(M)$, then

$$
\begin{aligned}
& \text { (i) }{ }^{*} \omega \in \Omega^{m-r}(M), \\
& \text { (ii) }{ }^{*}{ }_{m-r} *{ }_{r} \omega=(-1)^{p(m+1)} \omega .
\end{aligned}
$$

Definition 4.2. If $\omega, \vartheta \in \Omega_{\mathbf{R}}^{p}(M)$, we define their scalar product $(\omega, \boldsymbol{\vartheta}) \in \mathbf{R}$ as

$$
(\omega, \vartheta)=\int_{M} \omega \wedge * \vartheta
$$

If $\omega$ and $\vartheta$ are of different grades, we define $(\omega, \vartheta)=0$. Set $\|\omega\|_{0}^{2}=(\omega, \omega)$.

Proposition 4.3. If $\omega, \vartheta \in \Omega_{\mathbf{R}}^{p}(M)$, then

$$
\begin{aligned}
\text { (i) } & (\omega, \vartheta)=(\vartheta, \omega), \\
\text { (ii) } & (\omega, \omega)>0, \\
\text { (iii) } & (\omega, \omega)=0 \Rightarrow \omega=0, \\
\text { (iv) } & (\omega, \vartheta)=(* \omega, * \vartheta)
\end{aligned}
$$

Proof. Properties (i)-(iv) depend on local properties of forms on smooth manifolds.

Definition 4.3. If $\omega \in \Omega_{R}^{p}(M)$, we define $\delta \omega \in \Omega_{R}^{p-1}(M)$ and $\Delta \omega \in \Omega_{R}^{p}(M)$ by the formulas

$$
\begin{gathered}
\delta \omega=(-1)^{m(p+1)+1} * d * \omega, \quad m=\operatorname{dim} M, \\
\Delta \omega=(d \delta+\delta d) \omega .
\end{gathered}
$$

$\Delta$ is called the Laplace-Beltrami operator.

Proposition 4.4. For any $\omega, \vartheta \in \Omega_{\mathrm{R}}^{*}(M)$,

(i) $\quad(d \omega, \vartheta)=(\omega, \delta \vartheta)$,

(ii) $\quad(\Delta \omega, \vartheta)=(\omega, \Delta \omega) \quad((d+\delta) \omega, \vartheta)=(\omega,(d+\delta) \vartheta)$,

(iii) $(\Delta \omega, \omega)>0$, 
Proof. (i) follows from Stokes' formula, (4.2)(ii) and (4.4)(iv); (ii) and (iii) are consequences of (i).

\section{ChaPTER II. SMOOTHING PROBLEMS}

1. An analytical necessary and sufficient condition for smoothing. Suppose that $M^{m}$ is an $m$-dimensional PL-manifold. Let $\mathcal{T}=\left\{\Delta_{\alpha}^{r}\right\}_{\alpha \in \Lambda ; 0<r<m}$ be a triangulation of $M$ ( $r$ is the dimension of the closed simplex $\Delta_{\alpha}^{r}$ ), and let $\Gamma$ be a Riemannian structure of class $C^{0}$ on $M$.

Definition 1.1. If $x$ is any point in the triangulated PL-manifold $M$, we define

$$
\begin{aligned}
\Lambda(x) & =\left\{\alpha \mid \alpha \in \Lambda, x \in \Delta_{\alpha}^{r}, 0<r<m\right\}, \\
\Lambda^{\prime}(x) & =\left\{\alpha \mid \alpha \in \Lambda, x \in \Delta_{\alpha}^{r}, r=m\right\} .
\end{aligned}
$$

Let $T_{x}\left(\Delta_{\alpha}^{r}\right)$ denote the tangent space at $\Delta_{\alpha}^{r}$ in $x$.

Definition 1.2. If $M$ is a triangulated PL-manifold as above, let

$V_{\alpha}^{r}(x, M)=\left\{v \mid v=\dot{\lambda}(0) \in T_{x}\left(\Delta_{\alpha}^{r}\right), \lambda:[0,1] \rightarrow \Delta_{\alpha}^{r}, \lambda(0)=x, \lambda\right.$ of class $\left.C^{1}\right\}$.

The Riemannian structure in $M$ defines a flat metric on $V_{\alpha}^{r}(x, M)$. $V_{\alpha}^{r}(r, M)$ is called the tangent solid angle of $\Delta_{\alpha}^{r}$ in $x$. By definition, $\operatorname{dim} V_{\alpha}^{r}(x, M)=r$.

Definition 1.3. Let $\Delta_{\alpha}^{m}$ be a maximal simplex, and let $S_{\alpha, x}^{m-1}$ denote the unit sphere in $T_{x}\left(\Delta_{\alpha}^{m}\right)$ centered at 0 . Then

$$
\left|V_{\alpha}^{m}(x, M)\right|_{m}=\text { measure on } S_{\alpha, x}^{m-1} \text { of } V_{\alpha}^{m}(x, M) \cap S_{\alpha, x}^{m-1}
$$

is called the measure of the angle $V_{\alpha}^{m}(x, M)$.

The sum

$$
\Omega(x, M)=\sum_{\alpha \in \Lambda^{\prime}(x)}\left|V_{\alpha}^{m}(x, M)\right|_{m}
$$

is called the "volume density" of $\Gamma$ in $x$.

The incidence relations between the $\Delta_{\alpha}^{r}, \alpha \in \Lambda(x)$, determine incidence relations between the $V_{\alpha}^{r}(x, M), \alpha \in \Lambda(x)$; the latter determine incidence relations between the "spherical triangles":

$$
\tau_{\alpha}^{r-1}(x, M)=V_{\alpha}^{r}(x, M) \cap S_{\alpha, x}^{r-1}, \quad \alpha \in \Lambda(x) .
$$

DEFINITION 1.4. $\Sigma(x, M)$, and $V(x, M)$, are the complexes

$$
\begin{aligned}
& \Sigma(x, M)=\left\{\tau_{\alpha}^{r-1}\right\}_{\alpha \in \Lambda(x) ; 1<r<m}, \\
& V(x, M)=\left\{V_{\alpha}^{r}(x, M)\right\}_{\alpha \in \Lambda(x) ; 1<r<m} .
\end{aligned}
$$

Observation. $\Sigma(x, M) \subset V(x, M)$.

Definition 1.5. If $M^{m}, \mathcal{T}, \Gamma$ are as above, we say that $\Gamma$ has "constant volume density" iff, for any point $x \in M, \Omega(x, M)=\left|S^{m-1}\right|_{m-1}$.

We remark that the volume density is constant at every point off the $(m-2)$-skeleton. 
Proposition $1.1_{m}$. Let $\Gamma$ be a Riemannian metric of class $C^{0}$ on the manifold $M$ of dimension $m$. We suppose $\Gamma$ has constant volume density; then: (i) for any $x_{0} \in M$ there exists a PL-homeomorphism

$$
\varphi\left(x_{0}, M\right): V\left(x_{0}, M\right) \rightarrow \mathbf{E}^{m}, \quad \varphi\left(x_{0}, M\right)(0)=0,
$$

with the properties:

(ii) the homeomorphism $\varphi\left(x_{0}, M\right)$ restricted to any $V_{\alpha}^{r}\left(x_{0}, M\right) \alpha \in \Lambda\left(x_{0}\right)$, is an isometry on the image;

(iii) the homeomorphism $\varphi\left(x_{0}, M\right)$ which satisfies (ii) is unique up to an isometry of $\mathbf{E}^{m}$;

(iv) $\varphi\left(x_{0}, M\right)$ can be performed continuously with respect to $x_{0}$ on a stellar neighborhood of $x_{0}$.

From Proposition $1.1_{m}$ we deduce

THEOREM 1.2. If $\Gamma$ is a Riemannian metric with constant volume density of class $C^{0}$ on the $P L$-manifold $M$, then $\Gamma$ defines a differentiable structure of class $C^{1}$ on $M$, compatible with the combinatorial structure.

Proof. Set $V(M)=\cup_{x \in M} V(x, M)(U=$ disjoint union), endowed with the natural topology, and let $p: V(M) \rightarrow M$ be the map $p(V(x, M))=x$. Let $s: M \rightarrow V(M)$ be the map $s: x \mapsto 0 \in V(x, M)$. Then $\tau=(V(M), p, M, s)$ is evidently equivalent with the tangent microbundle of $M$ (see, e.g., Milnor [Mi]).

Property (ii) of Proposition $1.1_{m}$ enables us to introduce a vector space structure in the fibres of the tangent microbundle $\tau$. Property (iii) insures us that this vector space structure is well defined. Property (iv) expresses the local triviality of the fibration $\tau$. Hence $\mathcal{T}$ is a vector bundle. A theorem by Milnor [Mi] permits us to conclude that $M$ admits a well-defined differentiable structure of class $C^{1}$, compatible with the combinatorial structure of $M$.

REMARK. Property (ii) says that, if $\varphi(x, M)$ exists, it can be constructed as follows. We begin with a maximal solid tangent angle $V_{\alpha}^{m}(x, M)$ and we represent it isometrically in $\mathbf{E}^{m}$ in such a way that the vertex of $V_{\alpha}^{m}(x, M)$ goes into $0 \in \mathbf{E}^{m}$. Call this isometry $\varphi_{1}(x, M)$. We take another maximal solid tangent angle of $V(x, M)$ which is a neighbor to the first one; if $\varphi(x, M)$ exists, then $\varphi_{1}(x, M)$ can be extended at the new angle in a unique manner in order to verify (ii), and so on.

Proof of Proposition $1.1_{m}$. By induction on $m$. For $m=2$ the proposition is clearly true. We suppose we have proved Proposition $1.1_{m-1}$ and now we prove Proposition $1.1_{m}, m \geqslant 3$.

We suppose $x_{0} \in M$ is a vertex; if not, we perform a subdivision of $\tau$ in such a way that $x_{0}$ becomes a vertex (after the subdivision, $\Gamma$ still has constant volume density). 
Given that we have to verify a local property, we may restrict our considerations to $\operatorname{Star}\left(x_{0}, M\right)$; let $x_{0}, v_{1}, \ldots, v_{N}$ be all the vertices of $\operatorname{Star}\left(x_{0}, M\right)$. We can think of $\operatorname{Star}\left(x_{0}, M\right)$ as a part of $V\left(x_{0}, M\right)$. Any angle $V_{\alpha}^{r}\left(x_{0}, M\right)$ has a well-defined affine structure; let us denote the vectors $\overline{x_{0} v_{i}}$ by $X_{i}, i=1, \ldots, N$. If $v_{i} \in \Delta_{\alpha}^{r} \subset \operatorname{Star}\left(x_{0}, M\right)$, we consider on $\Delta_{\alpha}^{r}$ the field $\bar{X}_{i}$ of vectors parallel to $X_{i}$ and then we extend it on $V_{\alpha}^{r}(x, M) \supset \Delta_{\alpha}^{r}$.

The Riemannian metric $\Gamma_{\alpha}$ is determined by the scalar products $\left\langle\bar{X}_{i}, \bar{X}_{j}\right\rangle_{\xi}$, $\xi \in V_{\alpha}^{r}$.

LEMMA 1.1. With the same hypothesis and notations, we construct on $V\left(x_{0}, M\right)$ a constant Riemannian metric $\tilde{\Gamma}_{x_{0}}(M)=\left\{\tilde{\Gamma}_{\alpha}^{r}\right\}, \alpha \in \Lambda\left(x_{0}\right)$, given by the following scalar product:

$$
\left\langle\bar{X}_{i}, \bar{X}_{j}\right\rangle_{\xi}=\left\langle X_{i}, X_{j}\right\rangle_{x_{0}}, \quad \xi \in V\left(x_{0}, M\right) .
$$

Then $\tilde{\Gamma}_{x_{0}}(M)$ has constant volume density.

LEMMA 1.2. Let $V\left(x_{0}, M\right)$ be endowed with the Riemannian metric $\tilde{\Gamma}_{x_{0}}(M)$. Let $\tilde{\tilde{\Gamma}}_{x_{0}}\left(\Sigma\left(x_{0}, M\right)\right)$ be the induced Riemannian metric on $\Sigma\left(x_{0}, M\right) \subset V\left(x_{0}, M\right)$. Then $\tilde{\Gamma}_{x_{0}}\left(\Sigma\left(x_{0}, M\right)\right)$ has constant volume density.

We shall prove Lemmas 1.1 and 1.2 after completing the proof of Proposition $1.1_{m}$.

Suppose we have constructed $\varphi\left(x_{0}, M\right)$ satisfying Properties (i)-(iv). Let

$$
\Psi\left(x_{0}, M\right): \Sigma\left(x_{0}, M\right) \rightarrow \mathrm{S}^{m-1}
$$

be the restriction of $\varphi\left(x_{0}, M\right)$ to $\Sigma\left(x_{0}, M\right)$.

Let (i')-(iv') denote the statements (i)-(iv) from Proposition $1.1_{m}$ in which we have replaced $\varphi$ by $\Psi, V$ by $\tau$, and $\mathbf{E}$ by $S$. Then $\Psi\left(x_{0}, M\right)$ satisfies (i')-(iv').

Reciprocally, suppose we have a map $\Psi\left(x_{0}, M\right)$ which satisfies (i')-(iv'). Then there exists one and only one possibility in order to construct $\varphi\left(x_{0}, M\right)$ such that it satisfies (i)-(iv) and $\Psi=\varphi \mid \Sigma$. We shall indicate how $\Psi\left(x_{0}, M\right)$ can be constructed.

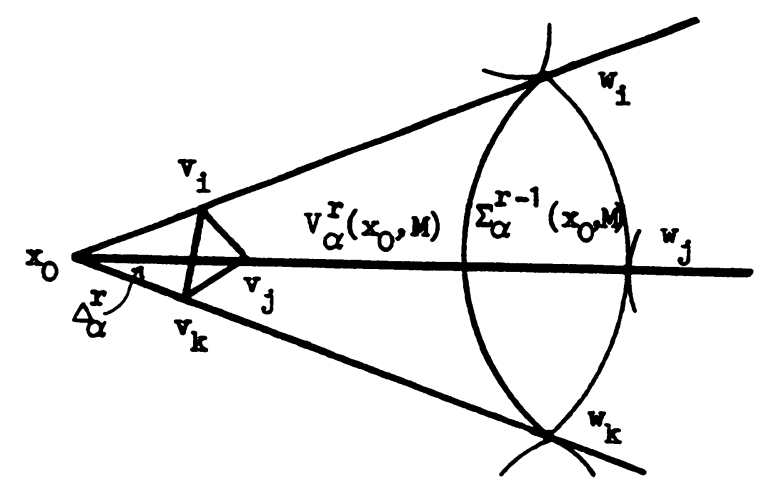


Let $w_{i}, 1<i \leqslant N$, be that vertex of $\Sigma\left(x_{0}, M\right)$ which is collinear with $x_{0}$ and $v_{i}$.

First we shall prove that we can construct $\Psi\left(x_{0}, M\right)$ on $\operatorname{Star}\left(w_{i}, \Sigma\right)$; afterwards, we shall show that $\Psi\left(x_{0}, M\right)$ can be extended over all $\Sigma\left(x_{0}, M\right)$.

Lemma 1.2. shows that $\tilde{\tilde{\Gamma}}_{x_{0}}\left(\Sigma\left(x_{0}, M\right)\right)$ has constant volume density; $\Sigma\left(x_{0}, M\right)$ being a combinatorial manifold of dimension $m-1$, we may apply Proposition $1.1_{m-1}$. Therefore there exists a PL-homomorphism

$$
\varphi_{0}\left(w_{i}, \Sigma\left(x_{0}, M\right)\right): V\left(w_{i}, \Sigma\left(x_{0}, M\right)\right) \rightarrow \mathbf{E}^{m-1}
$$

which satisfies (i)-(iv).

Let $\bar{w}_{i}$ be any point in $\mathbf{S}^{m-1}$. We have the diagram

$$
\begin{array}{ccc}
V\left(w_{i}, \Sigma\left(x_{0}, M\right)\right) & \varphi_{0}\left(w_{i}, \Sigma\left(x_{0}, M\right)\right) & \mathbf{E}^{m-1} \\
\exp _{w_{1} \downarrow} & & \downarrow \exp _{w_{i}} \\
\operatorname{Star}\left(w_{i}, \Sigma\left(x_{0}, M\right)\right) & \Psi_{w_{1}}\left(x_{0}, M\right) & \mathbf{S}^{m-1}
\end{array}
$$

where $\Psi_{w_{1}}\left(x_{0}, M\right)$ is that map which makes the diagram commutative, and the exponential mappings are taken with respect to the Riemannian metric $\tilde{\tilde{\Gamma}}_{x_{0}}\left(\left(\Sigma_{0}, M\right)\right)$ and the usual Riemannian metric on $\mathbf{S}^{m-1}$, respectively.

Observe that any 1-simplex $\left[w_{i}, w_{j}\right] \in \operatorname{Star}\left(w_{i}, \Sigma\left(x_{0}, M\right)\right)$ is an arc of the great circle.

The map $\Psi_{w_{i}}\left(x_{0}, M\right)$ has the following properties:

(1) it maps any 1 -simplex $\left[w_{i}, w_{j}\right] \in \operatorname{Star}\left(w_{i}, \Sigma\left(x_{0}, M\right)\right)$ into a congruent arc of a great circle in $\mathbf{S}^{m-1}$;

(2) it conserves the angle between any two 1 -simplices $\left[w_{i}, w_{j}\right],\left[w_{i}, w_{k}\right]$ belonging to the same 2-simplex contained in $\operatorname{Star}\left(w_{i}, \Sigma\left(x_{0}, M\right)\right)$;

(3) it is a homeomorphism from a neighborhood of $w_{i}$ to the image.

Therefore the map $\Psi_{w_{i}}\left(x_{0}, M\right)$ satisfies (i')-(ii').

Now we shall construct $\Psi\left(x_{0}, M\right)$. We fix a maximal simplex $\tau_{\alpha_{0}}^{m-1} \in$ $\Sigma\left(x_{0}, M\right)$, and we choose an isometric embedding:

$$
\Psi_{\alpha_{0}}\left(x_{0}, M\right): \tau_{\alpha_{0}}^{m-1} \rightarrow S^{m-1} .
$$

In order to define $\Psi\left(x_{0}, M\right)$, we will define its restrictions $\Psi_{\alpha}\left(x_{0}, M\right)=$ $\Psi\left(x_{0}, M\right) \mid \tau_{\alpha}^{m-1}$, where $\tau_{\alpha}^{m-1}$ is any maximal simplex of $\Sigma\left(x_{0}, M\right)$. There exists a chain $\gamma$ of maximal simplices:

$$
\gamma \equiv\left(\tau_{\alpha_{0}}^{m-1}, \tau_{\alpha_{1}}^{m-1}, \ldots, \tau_{\alpha}^{m-1}=\tau_{\alpha}^{m-1}\right)
$$

with the property that for any $i, 0<i<p-1, \operatorname{dim}\left(\tau_{\alpha_{i}}^{m-1} \cap \tau_{\alpha_{i+1}}^{m-1}\right)=m-2$.

Then there exists a unique continuous mapping

$$
\Psi_{\gamma}: \bigcup_{i=0}^{p} \tau_{\alpha_{i}}^{m-1} \rightarrow \mathbf{S}^{m-1}
$$


with the following properties:

(a) $\Psi_{\gamma} \mid \tau_{\alpha_{0}}^{m-1}=\Psi_{\alpha_{0}}\left(x_{0}, M\right)$,

(b) $\Psi_{\gamma}\left(\tau_{\alpha_{i}}^{m-1}\right) \cap \Psi_{\gamma}\left(\tau_{\alpha_{i+1}}^{m-1}\right)=\Psi_{\gamma}\left(\tau_{\alpha_{i}}^{m-1} \cap \tau_{\alpha_{i+1}}^{m-1}\right)$,

(c) $\Psi_{\gamma} \mid \tau_{\alpha_{1}}^{m-1}, 0 \leqslant i \leqslant p$, is an isometric embedding.

Condition (b) could require one to subdivide $\Sigma\left(x_{0}, M\right)$, which we suppose has been done. Now, $\Psi_{\gamma}$ can be constructed inductively starting with its definition on $\tau_{\alpha_{0}}^{m-1}$, then on $\tau_{\alpha_{1}}^{m-1}$, and so on.

By definition, we take $\Psi\left(x_{0}, M\right)\left|\tau_{\alpha}^{m-1}=\Psi_{\gamma}\right| \tau_{\alpha}^{m-1}$. It remains to check that this last restriction does not depend on the chain $\gamma$ connecting $\tau_{\alpha_{0}}^{m-1}$ with $\tau_{\alpha}^{m-1}$.

In order to prove it, notice that the induction hypothesis gives that for any simplex $\tau \in \Sigma\left(x_{0}, M\right)$ (of any dimension) and for any isometric embedding $\Psi_{\sigma}: \sigma \rightarrow \mathbf{S}^{m-1}$ of any maximal simplex $\sigma \in \operatorname{Star}\left(\tau, \Sigma\left(x_{0}, M\right)\right)$, there exists a unique embedding

$$
\Psi: \operatorname{Star}\left(\tau, \Sigma\left(x_{0}, M\right)\right) \rightarrow \mathbf{S}^{m-1}
$$

which: (i) extends $\Psi_{\sigma}$ continuously, and (ii) when restricted to any maximal simplex of $\operatorname{Star}\left(\tau, \Sigma\left(x_{0}, M\right)\right)$ is an isometry.

Notice also (this will be used below) that such a $\Psi$ is an open mapping (on the interior of $\left.\operatorname{Star}\left(\tau, \Sigma\left(x_{0}, M\right)\right)\right)$.

Correctness of the definition of $\Psi\left(x_{0}, M\right)$ depends also on the fact that $\mathbf{S}^{m-1}, m>3$, is simply connected.

Indeed, for any two chains $\gamma, \gamma^{\prime}$ connecting $\tau_{\alpha_{0}}^{m-1}$ and $\tau_{\alpha}^{m-1}$ there exists a "homotopy" $\gamma_{j}, 1<j \leqslant N$, i.e. a finite sequence of chains $\gamma_{j}$ with fixed ends: $\left(\gamma=\gamma_{1}, \gamma_{2}, \ldots, \gamma_{N}=\gamma^{\prime}\right)$ such that for any $j, 1<j \leqslant N-1$, the chains $\gamma_{j}$, $\gamma_{j+1}$ are of the form

$$
\gamma_{j} \equiv(a, b, c), \quad \gamma_{j+1} \equiv\left(a, b^{\prime}, c\right),
$$

where $(a),(b),\left(b^{\prime}\right),(c)$ denote chains and $b, b^{\prime}$ are contained in $\operatorname{Star}\left(\tau, \Sigma\left(x_{0}, M\right)\right)$, for an (arbitrary) simplex $\tau \in \Sigma\left(x_{0}, M\right)$.

From those considerations it follows also that $\Psi\left(x_{0}, M\right)$ is continuous. Indeed, let $\tau_{\alpha}^{m-1}, \tau_{\beta}^{m-1}$ be any two simplices, with $\operatorname{dim}\left(\tau_{\alpha}^{m-1} \cap \tau_{\beta}^{m-1}\right)=m-$ 2 , and let $\gamma$ be a chain connecting $\tau_{\alpha_{0}}^{m-1}$ with $\tau_{\alpha}^{m-1}$. Then $\left(\gamma, \tau_{\beta}^{m-1}\right)$ is a chain connecting $\tau_{\alpha_{0}}^{m-1}$ with $\tau_{\beta}^{m-1}$ and hence can be used to define $\Psi\left(x_{0}, M\right)$ on both $\tau_{\alpha}^{m-1}$ and $\tau_{\beta}^{m-1}$; from the definition of those restrictions it follows that $\Psi\left(x_{0}, M\right)$ is continuous on $\tau_{\alpha}^{m-1} \cap \tau_{\beta}^{m-1}$, and hence it is continuous.

As has been pointed out before, the induction hypothesis implies that $\Psi\left(x_{0}, M\right)$ is open. Therefore $\Psi\left(x_{0}, M\right)\left(\Sigma\left(x_{0}, M\right)\right)$ is open and closed in $\mathbf{S}^{m-1}$. Since $\mathbf{S}^{m-1}$ is connected, we have

$$
\Psi\left(x_{0}, M\right)\left(\Sigma\left(x_{0}, M\right)\right)=\mathbf{S}^{m-1} \text {. }
$$

$\Psi\left(x_{0}, M\right)$ being a local isometry, we have the strict inequality: 


$$
\text { Measure } \Psi\left(x_{0}, M\right)\left(\Sigma\left(x_{0}, M\right)\right)<\sum_{\tau_{\alpha^{m-1}} \in \Sigma\left(x_{0}, M\right)} \text { Measure } \Psi\left(x_{0}, M\right)\left(\tau_{\alpha_{1}}^{m-1}\right)
$$

if and only if $\Psi\left(x_{0}, M\right)$ is not injective. But the first term of the inequality is $\left|\mathbf{S}^{m-1}\right|$ because $\Psi\left(x_{0}, M\right)$ is surjective, while the second term is by hypothesis of Proposition $1.1_{m}$ just $\left|\mathbf{S}^{m-1}\right|$; hence $\Psi\left(x_{0}, M\right)$ is injective.

Now it is not hard to observe that $\Psi\left(x_{0}, M\right)$ satisfies all the required properties, and Proposition $1.1_{m}$ is proved.

Proof of Lemma 1.1. We have to check that $\langle$,$\rangle satisfies \Omega\left(\xi, V\left(x_{0}, M\right)\right)$ $=\left|\mathbf{S}^{m-1}\right|$ for any $\xi \in V\left(x_{0}, M\right)$.

Notice that it is enough to prove it for $\xi \in \Delta_{\alpha}^{r} \subset V\left(x_{0}, M\right)$. Notice also that the volume density at any point $\xi$ depends only on $\langle,\rangle_{\xi}$. We consider the following continuous deformation (indexed by the parameter $t \in[0,1]$ ) of the scalar product in $\xi$ :

$$
\left\langle\bar{X}_{i}, \bar{X}_{j}\right\rangle_{t, \xi}=\left\langle\bar{X}_{i}, \bar{X}_{j}\right\rangle_{(1-t) x_{0}+t \xi}
$$

We have

$$
\lim _{t>0}\langle,\rangle_{\xi, t}=\langle,\rangle_{x_{0}}=\langle,\rangle_{\xi}
$$

$\Omega(\xi, V(x, M))_{\langle,\rangle, \delta}$ is a continuous function of the variable $t$, and hence

$$
\begin{aligned}
\Omega\left(\xi, V\left(x_{0}, M\right)\right)_{\langle,\rangle \tau} & =\Omega\left(\xi, V\left(x_{0}, M\right)\right)_{\lim _{t>0},>\tilde{t}} \\
& =\lim _{t>0} \Omega\left(\xi, V\left(x_{0}, M\right)\right)_{\langle,\rangle_{(1-t) x_{0}+t \xi}} \\
& =\lim _{t>0}\left|S^{m-1}\right|=\left|S^{m-1}\right| .
\end{aligned}
$$

Since $\Gamma$ is a Riemannian metric with constant volume density, we have

$$
\Omega\left(\xi, V\left(x_{0}, M\right)\right)_{\langle,\rangle_{(1-t) x_{0}+t \xi}}=\Omega(\xi, M)_{<,>(1-\imath) x_{0}+t \xi}=\left|S^{m-1}\right|,
$$

for any $t \in(0,1]$. Therefore

$$
\Omega\left(\xi, V\left(x_{0}, M\right)\right)_{<, \tau \tau}=\lim _{t>0}\left|\mathbf{S}^{m-1}\right|=\left|\mathbf{S}^{m-1}\right|,
$$

which proves Lemma 1.1.

Proof of Leman 1.2. Let $\xi \in \Sigma\left(x_{0}, M\right)$ and let $V_{\alpha}^{r}\left(x_{0}, M\right)$ be any solid angle which contains $\xi$; then

$$
V_{\alpha}^{r}\left(\xi, V\left(x_{0}, M\right)\right)=V_{\alpha}^{r-1}\left(\xi, \tau_{\alpha}^{r-1}\left(x_{0}, M\right)\right) \oplus \mathbf{R} \xi
$$

$\oplus$ denoting orthogonal decomposition (observe that the ray which contains $\xi$ is orthogonal to the sphere $\left.S_{\alpha}^{r-1}\left(x_{0}, M\right)\right)$.

From this decomposition we deduce that

$$
\left|V_{\alpha}^{m}\left(\xi, V\left(x_{0}, M\right)\right)\right|=\frac{\left|\mathbf{S}^{m-1}\right|}{\left|\mathbf{S}^{m-2}\right|} \cdot\left|V_{\alpha}^{m-1}\left(\xi, \Sigma\left(x_{0}, M\right)\right)\right| \text {. }
$$


Therefore

$$
\begin{aligned}
\Omega\left(\xi, \Sigma\left(x_{0}, M\right)\right) & =\sum_{\alpha \in \Lambda^{\prime}\left(x_{0}\right)}\left|V_{\alpha}^{m-1}\left(\xi, \Sigma\left(x_{0}, M\right)\right)\right| \\
& =\frac{\left|\mathbf{S}^{m-2}\right|}{\left|\mathbf{S}^{m-1}\right|} \sum_{\alpha \in \Lambda^{\prime}\left(x_{0}\right)} \cdot\left|V_{\alpha}^{m}\left(\xi, V\left(x_{0}, M\right)\right)\right| \\
& =\frac{\left|\mathbf{S}^{m-2}\right|}{\left|\mathbf{S}^{m-1}\right|} \cdot\left|\mathbf{S}^{m-1}\right|=\left|\mathbf{S}^{m-2}\right|
\end{aligned}
$$

using Lemma 1.1 which completes the proof of Lemma 1.2.

2. Existence of a distance function implies smoothing. Let $M_{m}$ be a smooth manifold and let $\Gamma$ be a Riemannian structure on $M$, of class $C^{r}, r>3$.

A smooth function $r: M \times M \rightarrow[0, \infty)$ is called a distance function, cf. Hodge [Hd], iff $r$ has the following properties:

(i) $r(x, y)=r(y, x)>0$, for $x \neq y$,

(ii) $r(x, x)=0$,

(iii) $r^{2}(x, y)$ is of class $C^{2}$,

(iv) let $h: U \rightarrow \mathbf{R}^{m}$ be a coordinate chart on $U \subset M$ and let

$$
(h \times h)(x, y)=(h(x), h(y))=\left(x^{1}, \ldots, x^{m}, y^{1}, \ldots, y^{m}\right)
$$

for any $(x, y) \in U \times U$.

Then

$$
-\frac{1}{2} \frac{\partial^{2}}{\partial x^{i} \partial y^{j}} r^{2}(x, x)=g_{i j}(x),
$$

for any $x \in U$, where the $g_{i j}$ are the components of the fundamental tensor with respect to the coordinates $x^{1}, \ldots, x^{m}$.

Any smooth Riemannian manifold of class $C^{3}$ possesses at least one distance function. The geodesic distance is an example of a distance function. Any distance function can be used to construct a parametrix for the Laplacian (cf. Bidal-de Rham [B.R.]).

In this section we intend to define properly and to study the existence of distance functions on PL-manifolds with Riemannian structure.

By the end of this section, $M_{m}$ will denote a triangulated PL-manifold with Riemannian metric of class $C^{r}, r>3$.

Properties (i)-(iv) make sense even in this context. Only (iv) requires an explanation; in this case $U$ denotes any maximal closed simplex of the triangulation, and $h$ is any affine coordinate system on it.

Theorem 2.1. Let $M_{m}$ be a compact oriented PL-manifold. Then $M_{m}$ admits a Riemannian structure of class $C^{r}, r>3$, with at least one distance function if and only if $M_{m}$ is smoothable. 
Proof. Suppose $m \geqslant 3$; any manifold of dimension $<6$ admits a smooth structure; therefore this limitation does not restrict the generality of the theorem.

Let $\omega$ be the following double form on $M \times M$ (cf. Bidal-de Rham [B.R., p. 18], and de Rham [R, pp. 143-144]):

$$
\omega(x, y)=\frac{1}{(m-2)\left|\mathbf{S}^{m-1}\right| r^{m-2}} \sum_{p=0}^{m} \frac{1}{p !}\left[d_{x} d_{y}\left(-\frac{1}{2} r^{2}(x, y)\right)\right]^{p},
$$

where $\left|\mathbf{S}^{m-1}\right|$ denotes the measure of the unit sphere in $\mathbf{E}^{m}$. In the smooth case, the form $\omega$ would be a parametrix for the Laplacian.

Theorem 2.1 will follow from Lemmas 2.1 and 2.2.

LEMMA 2.1. For any form $\phi$ on $M$ of class $C^{r}, r>2$, the left-hand side of (2.3) is a form of class $C^{0}$ on $M$.

LEMMA 2.2. For any $\phi$ on $M$ of degree $p, 0<p<m$, and class $C^{r}, r>2$, the following identity holds:

$$
\int_{M(y)} \omega(x, y) \wedge *{ }_{y} \Delta_{y} \phi(y)-\Delta_{y} \omega \wedge *{ }_{y} \phi(y)=\mu(x) \phi(x)
$$

where $\mu(x)$ is the volume density of the Riemannian metric $\Gamma$ at $x$ (see $\$ 1$ of Chapter II).

Suppose we have proved these lemmas. Let us take $\phi \equiv 1$ in (2.3). Then we deduce that $\mu(x)$ is a continuous function on $M$. But $\mu(x)=\left|S^{m-1}\right|$ for any interior point $x$ of any maximal simplex of the triangulation and hence, by the continuity of $\mu$ we deduce that the Riemannian metric $\Gamma$ has constant volume density. Theorem 1.2 says that $M$ is smoothable. If $M$ is smoothable, then there exists a distance function, as we have explained above. Theorem 2.1 is proved.

REMARK 2.1. We have used only the component of degree 0 of $\omega$ in order to prove Theorem 2.1.

Let us consider Lemma 2.2 first.

Lemma 2.2 is a translation, from the smooth case into the combinatorial case, of Formula I of Bidal-de Rham [B.R., p. 18] or of Formula I of de Rham [R, p. 146]. For its proof we shall use Bidal-de Rham [B.R.].

The first part of the proof (Green formula) can be carried out without any modification. Let $B \subset M$ be a small combinatorial ball which contains $x \in M$. As the integrand is regular on $M-B$, the Green formula gives us

$$
\begin{aligned}
\int_{M-B} \omega \wedge & * \Delta \phi-\Delta \omega \wedge * \phi \\
& =-\int_{\partial B} \phi \wedge * d \omega-\delta \omega \wedge * \phi+\delta \phi \wedge * \omega-\omega \wedge * d \phi,
\end{aligned}
$$


and hence

$$
\begin{aligned}
\int_{M} \omega \wedge & * \Delta \phi-\Delta \omega \wedge * \phi \\
& =\lim _{|B| \rightarrow 0} \int_{\partial B} \phi \wedge * d \omega-\delta \omega \wedge * \phi+\delta \phi \wedge * \omega-\omega \wedge * d \phi,
\end{aligned}
$$

where $|B|$ is the measure of $B ;|B| \rightarrow 0$ means we take a family of homothetic balls $\varepsilon B$, with $\varepsilon \rightarrow 0$.

From now on, the proof is based on estimates of $\omega, d_{y} \omega, \delta_{y} \omega, \Delta_{y} \omega$, near the diagonal. These estimates can be obtained just as in the smooth case. Indeed, in both the smooth case and the combinatorial case, it is necessary to study these forms at points $(x, y) \in M \times M$, with $x$ fixed and $y$ near $x$. This observation allows us to focus our attention, separately, on each maximal simplex of $M$ which contains $x$; so this is the same problem as in the smooth case.

The estimates are obtained by the following method. First, one approximates the Riemannian metric $g_{i j}$ (on a neighborhood of $x$ ) by a flat one $\bar{g}_{i j}$ which has contact of order 1 at $x$ (it is called osculator), i.e.,

$$
\bar{g}_{i j}(x)=g_{i j}(x), \quad\left(\frac{\partial}{\partial x^{k}} \bar{g}_{i j}\right)(x)=\left(\frac{\partial}{\partial x^{k}} g_{i j}\right)(x),
$$

then one approximates the distance function $r$ by the distance function $\bar{r}$, the Euclidean distance given by $\bar{g}_{i j}$. A function $f(y)$ is said to be of order $k$ on a neighborhood of $x$ (see [B.R., p. 24]) if $f(y) / r^{k}(x, y)$ is bounded on a small neighborhood of $x$. One obtains the following estimates:

(i) $r^{2}(x, y)-\bar{r}^{2}(x, y)$ is of order 3 ;

(ii) $g_{i j}-\bar{g}_{i j}$ is of order 2 .

Let us denote $g=\operatorname{det}\left(g_{i j}\right), \bar{g}=\operatorname{det}\left(\bar{g}_{i j}\right)$. Then, it follows from (ii) that:

(iii) $g-\bar{g}, \sqrt{g}-\sqrt{\bar{g}}, g^{i j}-\bar{g}^{i j}$ are of order 2 .

Let $₹$ denote the *-operator with respect to the metric $\bar{g}$; let us put $\bar{\delta}= \pm \bar{*} \bar{*}$.

Then one obtains the following estimates:

(iv) if $\alpha$ is of order $k$, then

(a) $* \alpha-\bar{*} \alpha$ is of order $k+2$,

(b) $\delta \alpha-\bar{\delta} \alpha$ is of order $k+1$,

(c) $\Delta \alpha-\bar{\Delta} \alpha$ is of order $k$.

Let $\bar{\omega}$ denote the analogue of $\omega$ which arises from $\bar{r}$. It is very easy to determine the orders of $\bar{\omega}, \bar{d} \bar{\omega}, \bar{\delta} \bar{\omega}, \bar{\Delta} \bar{\omega}$. From these estimates and the estimates (i)-(iv), one can deduce the estimates

(v) $\omega(x, y)$ is of order $2-m$,

(vi) $* d_{y} \omega(x, y)-\bar{*} d_{y} \bar{\omega}(x, y)$, and $\delta_{y} \omega(x, y)-\bar{\delta}_{y} \bar{\omega}(x, y)$ are of order $3-$ $m$, 
(vii) $\Delta_{y}(x, y)$ is of order $2-m$.

The following remark will be useful later.

REMARK 2.2. Suppose we take instead of the osculator metric $\bar{g}$ a new metric $\bar{g}^{\prime}$ which has only a contact of order 0 at $x$, i.e., $g_{i j}(x)=\bar{g}_{i j}^{\prime}(x)$. Then all estimates (i)-(vi) remain valid if the order of the estimates is reduced by 1 .

From (v) we deduce

$$
\lim _{|B| \rightarrow 0} \int_{\partial B} \delta \phi \wedge * \omega-\omega \wedge * d \phi=0
$$

and hence (2.5) becomes

$$
\int_{M} \omega \wedge * \Delta \phi-\Delta \omega \wedge * \phi=-\lim _{|B| \rightarrow 0} \int_{\partial B} \phi \wedge * d \omega-\delta \omega \wedge * \phi .
$$

It remains to prove that the right-hand side (R.H.S.) of $(2.6)$ is $\mu(x) \cdot \phi(x)$. At this point, we remark that the Bidal-de Rham method may no longer be applied directly. Indeed, they observe that if $\theta$ is a form of order $2-m$, then

$$
\lim _{|B| \rightarrow 0} \int_{\partial B} \theta=0
$$

and hence, by (vi), $\omega$ may be changed to $\bar{\omega}$ in the R.H.S.

Successively, Bidal-de Rham [B.R.] use the Stokes formula twice and the result follows.

Unfortunately, in the combinatorial case we do not know whether there exists an osculator metric $\bar{g}$ of class $C^{3}$ on $\operatorname{Star}(x, M)$. Hence, if we want to apply the Stokes formula, the simplex-by-simplex approximation of the $g_{i j}$ by $\overline{\boldsymbol{g}}_{i j}$ is no longer useful.

Fortunately, there exists a Riemannian metric $\bar{g}^{\prime}$ of class $C^{\infty}$ on $\operatorname{Star}(x, M)$, which has a contact of order 0 with $g$ on each simplex of $\operatorname{Star}(x, M)$.

Indeed, the "constant Riemannian metric" (see Lemma 1.1) which extends the Riemannian metric $g(x)$ has this property.

Let us apply, at this point, the estimate (vi) along with Remark 2.2. Then, for each $m$-simplex $\sigma \in \operatorname{Star}(x, M)$, we have

$$
\lim _{|B| \rightarrow 0} \int_{\partial B \cap \sigma} \phi \wedge * d \omega-\delta \omega \wedge * \phi=\lim _{|B| \rightarrow 0} \int_{\partial B \cap \sigma} \phi \wedge \bar{*}^{\prime} d \bar{\omega}^{\prime}-\overline{\delta^{\prime}} \bar{\omega}^{\prime} \wedge{\overline{*^{\prime}} \phi}^{\prime}
$$

where $\bar{\omega}^{\prime}, \overline{\boldsymbol{F}}^{\prime}, \bar{\delta}^{\prime}$, are, respectively, the form and the operators which arise from $\overline{\mathbf{g}}^{\prime}$.

The right-hand side of (2.7) does not change if we take instead of $\phi$ the constant form $\tilde{\phi}$, where $\tilde{\phi}(y)=\phi(x)$, for any $y \in \sigma \cap B$.

Hence

$$
\text { R.H.S. }=-\lim _{|B| \rightarrow 0} \int_{\partial B} \tilde{\phi} \wedge \overline{\bar{F}^{\prime}} d{\overline{\omega^{\prime}}}^{\prime}-\overline{\delta^{\prime} \bar{\omega}^{\prime}} \wedge \overline{\bar{F}^{\prime}} \tilde{\phi}
$$


From now on the method of Bidal-de Rham may be used, and the result follows.

Lemma 2.1 follows from the estimates (v), (vii).

3. Smoothing problems. Applications. In this section we intend to present some applications of Proposition 1.1 and Theorem 1.2.

Let $\phi:\left(\mathbf{R}^{m}, 0\right) \rightarrow\left(\mathbf{E}^{m}, 0\right)$ be a PL-homeomorphism. There exist a triangulation $D$ of $\mathbf{R}^{m}$ and a triangulation $E$ of $\mathbf{E}^{m}$ such that $\phi$ is simplicial; we can arrange $D$ and $E$ such that the origins are vertices in $D$ and $E$.

Let us consider $\operatorname{Star}(0, D)$. Let us take off all vertices, except 0 , of $D$ and $E$, and let us prolong radially from 0 all simplices which contain 0 of $\operatorname{Star}(0, D)$ (resp., $\operatorname{Star}(0, E)$ ). Thus, we obtain a decomposition $\bar{D}$ (resp., $\bar{E}$ ) of $\mathbf{R}^{m}$ (resp., $\left.\mathbf{E}^{m}\right)$ by "simplicial cones" with vertex at 0 .

The mapping $\phi \mid \operatorname{Star}(0, D)$ extends linearly, in a unique manner, to all $\mathbf{R}^{n}$ and it remains a PL-homeomorphism. Let us denote by $\bar{\phi}$ the new PL-homeomorphism. The associate mapping $\bar{\phi}$ has the following property: let $\phi_{1}, \phi_{2}$ be two PL-homeomorphisms from $\mathbf{R}^{m}$ into $\mathbf{E}^{m}$; then $\bar{\phi}_{1} \equiv \bar{\phi}_{2}$ if and only if germ $_{0} \phi_{1}=$ germ $_{0} \phi_{2}$.

Let $\phi$ be as above. Let $\Gamma_{\phi}$ be the Riemannian metric on $\mathbf{R}^{m}$ :

$$
\Gamma_{\phi}=\phi^{*}(\eta),
$$

where $\eta$ is the Euclidean metric in $\mathbf{E}^{m}$.

The Riemannian metric $\Gamma_{\phi}$ is determined by its restriction at 0 , because $\Gamma_{\phi}$ is "constant" on each simplicial cone of the decomposition. Moreover, $\Gamma_{\phi}$ has constant volume density.

Reciprocally, suppose we have a decomposition of $\mathbf{R}^{m}$ into simplicial cones with vertices at 0 ; a metric $g$ at $0 \in \mathbf{R}^{m}$ is by definition an assignment $\sigma \mapsto g_{\sigma}$ which associates with any maximal closed cone $\sigma$ a scalar product $g_{\sigma}$ on the tangent space $T_{0} \sigma$ such that for any two maximal cones $\sigma, \tau$, the scalar products $g_{\sigma}, g_{\tau}$ agree on the common tangent vectors. If $g$ is a metric at $0 \in \mathbf{R}^{m}$, we can extend it over all $\mathbf{R}^{m}$ as in Lemma 1.1. If this extended Riemannian metric has constant volume density, we say that the initial metric $g$ at 0 is "normalized". Hence, a Riemannian metric with constant volume density over $M$ is nothing but a field on $M$ of normalized metrics.

Definition 3.1. Let $\Gamma(m)$ denote the set of all normalized metrics at $0 \in \mathbf{R}^{m}$ with respect to all possible decompositions of $\mathbf{R}^{m}$ in simplicial cones.

Now we shall indicate how to introduce a topology on $\Gamma(m)$.

Let $D$ be any decomposition of $\mathbf{R}^{m}$ in simplicial cones with vertex in 0 . For any 1-dimensional simplicial cone of the decomposition $D$, let us choose a point on it, different from the vertex 0 ; let $e_{1}, \ldots, e_{p}$ be these points, which we think of as vectors. Any normalized metric in 0 is determined by the matrix with real entries $\left\{\left(e_{i}, e_{j}\right)\right\}_{i, j} \in \mathbf{R}^{N}$, where $i, j$ are such that $e_{i}, e_{j}$ belong to a 2-dimensional simplicial cone of $D$. Therefore any normalized metric at 0 
can be thought of as a point of $\mathbf{R}^{N}$, where $N$ is the cardinal of the set of all 2-dimensional simplicial cones of $D$. In other words, the set of all normalized metrics at $0 \in \mathbf{R}^{m}$, constructed on the decomposition $D$, can be endowed with a topology, that induced by $\mathbf{R}^{N}$.

Let $\Gamma(D)$ denote this topological space. If $D^{\prime}$ is a refinement of the decomposition $D$ (so that $D^{\prime}$ is still a decomposition by simplicial cones), then $\Gamma(D)$ can be thought of as a subspace of $\Gamma\left(D^{\prime}\right)$.

As

$$
\tilde{\Gamma}(m)=\lim _{\vec{D}} \Gamma(D) \quad \text { (as sets), }
$$

we shall introduce on $\tilde{\Gamma}(m)$ the weak topology, i.e., $U \subset \tilde{\Gamma}(m)$ is by definition open in $\tilde{\Gamma}(m)$ iff $U \cap \Gamma(D)$ is open in $\Gamma(D)$ for any $D$.

Let PL $(m)$ denote the group of germs of PL-homeomorphisms $\Phi:\left(\mathbf{R}^{m}, 0\right)$ $\rightarrow\left(\mathbf{E}^{m}, 0\right)$. The space

$$
\Gamma(m)=\operatorname{PL}(m) / O(m)
$$

is fundamental in the theory of smoothing.

THEOREM 3.1. Let $\phi$ be the germ of a PL-homeomorphism $\phi:\left(\mathbf{R}^{m}, 0\right) \rightarrow$ $\left(\mathbf{E}^{m}, 0\right)$. Then the correspondence

$$
\Psi: \operatorname{PL}(m) \rightarrow \tilde{\Gamma}(m), \quad \Psi: \phi \mapsto \Gamma_{\phi}
$$

factorizes to a bijection

$$
\Xi: \Gamma(m) \rightarrow \tilde{\Gamma}(m)
$$

Proof. The map $\Psi$ factorizes because an orthogonal transformation of $\mathbf{E}^{m}$ does not change the metric of $\mathbf{E}^{m}$.

The proof of Proposition 1.1 shows that for any normalized metric $\gamma$ at $0 \in \mathbf{R}^{m}$, there exists a PL-homeomorphism $\phi:\left(\mathbf{R}^{m}, 0\right) \mapsto\left(\mathbf{E}^{m}, 0\right)$ such that $\Gamma_{\phi}$ is just the Riemannian metric $\gamma$. Hence $\Xi$ is an epimorphism.

Suppose the $\phi_{i}:\left(\mathrm{R}^{m}, 0\right) \rightarrow\left(\mathrm{E}^{m}, 0\right), i=1,2$, are two PL-homeomorphisms such that $\Gamma_{\phi_{1}}=\Gamma_{\phi_{2}}$. The proof of Proposition 1.1 shows again that $\phi_{1}, \phi_{2}$ differ by an orthogonal transformation of $\mathbf{E}^{m}$, which proves injectivity.

TheOREM 3.2. Let $M_{m}$ be a PL-manifold. Let $x$ be any point of $M_{m}$ and let $\Gamma_{x}$ denote the (topological) space of all normalized metrics in $x$. Then $\Gamma(M)=$ $\bigcup_{x \in M} \Gamma_{x}$ is the total space of a locally trivial fibre bundle over $M$, with fibre $\Gamma(m)$, such that any Riemannian structure with constant volume density on $M$ is a continuous section in this bundle.

From Theorems 1.2 and 3.2 we deduce the following corollaries:

COROLLARY 3.1. The combinatorial manifold $M$ admits a differential structure if and only if there exists a continuous global section in $\Gamma(M)$. Any continuous section in $\Gamma(M)$ defines a smoothing. 
Corollary 3.2. Any two homotopic sections in $\Gamma(M)$ define concordant smoothings.

COROLlaRY 3.3. There exists an obstruction theory for smoothing.

Results of this nature are well known in the theory of smoothing, but the definition of the associated bundle $\Gamma(M)$ is different. Such results are due to J. Munkres, R. Thom, M. Hirsch, and J. Milnor.

\section{Chapter III. COMBINATORIAL Hodge THEORY}

1. Distributions. Throughout this chapter, $M^{m}$ denotes a compact oriented combinatorial manifold without boundary of dimension $m$.

For any maximal simplex $\sigma \in M$ we choose a linear embedding $\rho_{\sigma}$ of $\sigma$ in $\mathbf{R}^{m} ; \rho_{\sigma}$ is a coordinate chart on $\sigma$, and let us denote these coordinates by $x_{\sigma}$.

Let $\Gamma$ be any $C^{\infty}$ Riemannian metric on $M^{m}$ such that for any $\sigma \in M$, all the components of $\Gamma_{\sigma}$ (with respect to the coordinates $x_{\sigma}$ ) are constant functions. There exists at least one such Riemannian metric. Indeed, it is enough to embed $M$ linearly in $\mathbf{E}^{N}$ and take as $\Gamma$ the induced Riemannian metric.

As in the case of smooth manifolds (see, e.g., de Rham [R] or Schwartz [Sc]) we shall introduce:

Defintron 1.1. A linear functional $T: \Omega^{p}(M) \rightarrow \mathbf{R}$ will be called a distributional form (or distribution) of degree $m-p$ on $M$ iff there exist a natural $N$ (which depends on $T$ ) and a positive constant $C$, such that

$$
|T \omega| \leqslant C \operatorname{Sup}\left|D_{x_{0}}^{\alpha} \omega_{o}(x)\right|, \quad \omega \in \Omega^{p}(M),
$$

where the supremum is taken with respect to:

(a) any partial derivative $D_{x_{0}}^{\alpha},|\alpha| \leqslant N$,

(b) any maximal simplex $\sigma \in M$ and $x \in \sigma$,

(c) any $I=\left(i_{1}, \ldots, i_{p}\right), \omega_{\sigma, I} d x^{I}=\omega_{0}$.

The set of all distributions of degree $m-p$ will be denoted by $\mathscr{Q}^{m-p}$.

As in the smooth case, any form $\vartheta \in \Omega^{p}$ or any chain $\gamma$ of degree $m-p$ defines a distribution of degree $p$ :

$$
\begin{aligned}
& \vartheta(\omega)=\int \vartheta \wedge \omega, \quad \omega \in \Omega^{m-p}, \\
& \gamma(\omega)=\int_{\gamma} \omega .
\end{aligned}
$$

If $T \in \mathscr{Q}^{p}$ and $\vartheta \in \Omega^{q}(M)$ is a form, their exterior product $T \wedge \vartheta \in \mathscr{Q}^{p+q}$ is defined as

$$
(T \wedge \vartheta)(\omega)=T(\vartheta \wedge \omega), \quad \omega \in \Omega^{m-p-q} .
$$

If $f \in \Omega^{0}(M)$, and $T \in \mathscr{T}^{m-p}, f T \in \vartheta^{m-p}$ and

$$
(f T)(\omega)=T(f \omega) .
$$

Hence $\mathscr{Q}^{p}$ is a module over $\Omega^{0}(M)$. 
If $T \in \mathscr{Q}^{p}$, we define $d T \in \mathscr{Q}^{p+1}$ :

$$
(d T)(\omega)=(-1)^{p} T(d \omega), \quad \omega \in \Omega^{m-p-1},
$$

and the usual formula holds:

$$
d(T \wedge \vartheta)=(d T) \wedge \vartheta+W T \wedge d \vartheta
$$

where $W T=(-1)^{p} T$ for $T \in \mathscr{Q}^{p}$. It is clear that $d^{2}=0$.

We can define, as usual, distributional forms on any open set $U \subset M$; it makes sense to restrict any distributional form in $\mathscr{Q}^{p}$ to any open set $U$. These constructions will permit us to speak about the support of a distribution and about germs of distributional forms $\omega \in \mathscr{Q}^{p}$. The germs of distributional forms in $\mathscr{Q}^{p}$ can be organized as a sheaf and we shall indicate it by $\mathscr{Q}^{p}$.

2. Sobolev spaces. We shall introduce on $\Omega^{*}(M)$ various scalar products in order to construct a Rellich chain of Hilbert spaces on $M$.

We define, for any $s>0$,

$$
\begin{aligned}
(\alpha, \beta)_{s} & =\left(\alpha,(1+\Delta)^{s} \beta\right), \quad \alpha, \beta \in \Omega^{*}(M), \\
\|\alpha\|_{s}^{2} & =(\alpha, \alpha)_{s} .
\end{aligned}
$$

Let $\nabla_{i}$ denote the covariant derivative $\nabla_{i}=\nabla \partial / \partial x_{\sigma}^{i}$.

We can define also new scalar products on $\Omega^{*}(M)$ :

$$
(\alpha, \beta)_{s}^{\hat{s}}=\sum_{\substack{0<k<s \\
\sigma}}\left(\begin{array}{l}
s \\
k
\end{array}\right) \int_{\sigma} g^{i_{1} j_{1}} \cdots g^{i_{k} j_{k}} \nabla_{i_{1}} \cdots \nabla_{i_{k}} \alpha \wedge * \nabla_{j_{1}} \cdots \nabla_{j_{k}} \beta
$$

where $\sigma$ runs over all maximal simplices of $M$.

If $\alpha, \beta$ in (2.1) and (2.2) are homogeneous, but of different degrees, we define $(\alpha, \beta)_{s}=(\alpha, \beta)_{s}^{n}=0$.

REMARK 2.1. Both $(,)_{s}$ and $(,)_{s}$ are symmetric and positive definite.

The scalar product $(,)_{s}$ depends only on the metric $\Gamma$.

Proposition 2.1. For any $s=0,1, \ldots$, and for any $\alpha, \beta \in \Omega^{*}(M)$,

$$
(\alpha, \beta)_{s}=(\alpha, \beta)_{s} \text {. }
$$

Proof. Let $s=1$. We have

$$
\begin{aligned}
(\alpha, \Delta \beta) & =-\sum_{\sigma \in M} \int_{\sigma} g^{i j} \alpha_{\sigma} \wedge^{*} \nabla_{i} \nabla_{j} \beta_{\sigma} \\
& =\sum_{\sigma \in M} \int_{\sigma} g^{i j} \nabla_{i} \alpha_{\sigma} \wedge^{*} \nabla_{j} \beta_{\sigma}-\sum_{\sigma \in M} \int_{\sigma} \nabla_{i}\left(g^{i j} \alpha_{\sigma} \wedge^{*} \nabla_{j} \beta_{\sigma}\right) .
\end{aligned}
$$

We will prove that the last sum vanishes.

Let $\left\{\theta_{\sigma}\right\}_{\sigma}=\theta$ denote the 1-form on $M$ (see Tanaka [T, p. 50]):

$$
\theta_{\sigma}(x)=*\left[\alpha_{0} \wedge * \nabla_{x} \beta_{o}\right]
$$

$x$ being any tangent vector to $M^{(m)}-M^{(m-2)} \cdot \theta$ is a $C^{\infty}-$ form on $M$. Then 
we have

$$
\delta \theta=-g^{i j} \frac{\partial}{\partial x^{i}} \theta_{j}
$$

where

$$
\theta_{j}=\theta\left(\frac{\partial}{\partial x^{j}}\right)=*\left[\alpha \wedge * \nabla_{j} \beta\right]
$$

it follows that

$$
\delta \theta=-g^{i j} \nabla_{i} *\left[\alpha \wedge * \nabla_{j} \beta\right]
$$

or

$$
d * \theta=\nabla_{i} g^{i j}\left[\alpha \wedge * \nabla_{j} \beta\right]
$$

therefore

$$
\sum_{\sigma \in M} \int_{\sigma} \nabla_{i}\left(g^{i j} \alpha_{\sigma} \wedge * \nabla_{j} \beta_{\sigma}\right)=\int_{M} d * \theta=\int_{\partial M=\varnothing} * \theta=0 .
$$

Now, (2.3), for $s=1$, follows from (2.4) and (2.5), because we have

$$
(\alpha, \Delta \beta)_{0}=\sum_{\sigma \in M} \int_{\sigma} g^{i j} \nabla_{i} \alpha \wedge * \nabla_{j} \beta .
$$

In order to prove (2.3) for an arbitrary $s$, it is enough to iterate (2.6):

$$
\left(\alpha, \Delta^{s+1} \beta\right)_{0}=\left(\alpha, \Delta\left(\Delta^{s} \beta\right)\right)_{0}
$$

Definition 2.1. The completion of the pre-Hilbert space $\Omega^{p}(M)$ with respect to the norm \|\|$_{s}$ (or \|\|$_{s}^{\wedge}$ ) will be called the Sobolev space of order $s$, and it will be denoted by $H_{s}^{P}(M)$.

Set $H_{s}^{*}(M)=\bigoplus_{p} H_{s}^{p}(M)$. We have the following analog of the Rellich theorem.

THEOREM 2.1. For any $s>0$, the identity $\Omega^{*}(M) \stackrel{i}{\rightarrow} \Omega^{*}(M)$ extends to a continuous homomorphism

$$
j(s, s+1): H_{s+1}^{*}(M) \rightarrow H_{s}^{*}(M) .
$$

The map $j(s, s+1)$ is injective, completely continuous, and the image of $H_{s+1}$ in $H_{s}$ is dense.

ProOF. Let $\Omega^{*}(\sigma)$ denote the algebra of real smooth forms over the simplex $\sigma$; we consider $\Omega^{*}(\sigma)$ endowed with the norm \|\|$_{s}$, (2.2). The metric $\Gamma$ being flat, the norm \|\|$_{s}$ is the usual Sobolev norm. We have the natural mapping

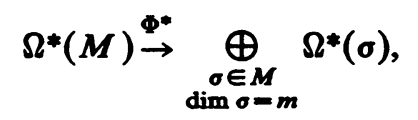

and we introduce in $\bigoplus_{\sigma} \Omega^{*}(\sigma)$ the direct sum norm: for $\omega=\left(\omega_{\sigma}\right)_{\sigma} \in$ $\bigoplus_{0} \Omega^{*}(\sigma)$, we set 


$$
\|\sigma\|_{s}^{2}=\sum_{\sigma}\left\|\omega_{\sigma}\right\|_{s}^{2}
$$

Then, for any $s>0$, we deduce from (2.2), (2.8) and (2.9) that

$$
\left(\Omega^{*}(M),\|\|_{s}\right) \stackrel{\Phi_{s}^{*}}{\rightarrow}\left(\bigoplus_{\sigma} \Omega^{*}(\sigma),\|\|_{s}^{i}\right)
$$

in an injective isometry.

When we complete $\Omega^{*}(M)$ and $\bigoplus_{0} \Omega^{*}(\sigma)$ with respect to the Sobolev norms \|\|$_{s}$, the induced map $\Phi_{S}^{*}$ remains injective. We observe that

$$
\overline{\bigoplus_{\sigma} \Omega^{*}(\sigma)_{\|_{s}}}=\bigoplus_{\sigma} \overline{\Omega^{*}(\sigma)_{\|\|_{s}}}=\bigoplus_{\sigma} H_{s}^{*}(\sigma) \text {. }
$$

where $\|_{s}$ denotes completion with respect to the norm \|\|$_{s}$, while $H_{s}^{*}(\sigma)$ denotes the Sobolev space of order $s$ of *-forms. Therefore we have the isometric injection

$$
\hat{\Phi}_{s}^{*}: H_{s}^{*}(M) \rightarrow \bigoplus_{\sigma} H_{s}^{*}(\sigma)
$$

Consider the commutative diagram

$$
\begin{array}{ccc}
H_{s+1}^{*}(M) & \stackrel{\hat{\Phi}_{s+1}^{*}}{\rightarrow} & \bigoplus_{\sigma} H_{s+1}^{*}(\sigma) \\
\downarrow j(s, s+1) & & \downarrow \bigoplus_{\sigma} j_{\sigma}(s, s+1) \\
H_{s}^{*}(M) & \stackrel{\hat{\Phi}^{*}}{\rightarrow} & \bigoplus H_{s}^{*}(\sigma) .
\end{array}
$$

The Rellich theorem in $\sigma$ (see, e.g., Agmon [A]) says that $j_{\sigma}(s, s+1)$ is completely continuous; it follows that even $\bigoplus_{\sigma} j(s, s+1)$ is completely continuous. This property, together with the fact that $\Phi_{s+1}^{*}$ and $\Phi_{s}^{*}$ are isometric injections, implies that $j(s, s+1)$ is completely continuous.

The $j_{\sigma}(s, s+1)$ are injective (see, e.g., Agmon [A]); therefore $\bigoplus_{\sigma} j(s, s+$ $1)$ is injective; hence from the diagram we deduce that $j(s, s+1)$ is injective.

$H_{s+1}^{*}$ is evidently dense in $H_{s}^{*}(M)$ because $\Omega^{*}(M)$ is dense in both of them, by definition.

Definition 2.2. For any $s \geqslant 0$, define $H_{-s}^{p}$ to be the dual of the Hilbert space $H_{s}^{p}$, or

and

$$
H_{-s}^{p}(M)=\left(H_{s}^{p}(M)\right)^{*}, \quad 0<p<m
$$

$$
j(-s,-s-1)=j(s, s+1)^{*}: H_{-s}^{p}(M) \rightarrow H_{-s-1}^{p}(M) .
$$

THEOREM 2.2. The statement of Theorem 2.1 is still valid even for $s<0$.

Proof. See Palais [P, Chapter VII, §1].

COROLlARY $2.2^{\prime}$. The sequence of Hilbert spaces and maps

$$
j(s, s+1): H_{s+1}^{*}(M) \rightarrow H_{s}^{*}(M), \quad s \in \mathbf{Z},
$$

is a discrete Rellich chain. 
Proposition 2.3. If $\omega \in H_{s}^{P}(M)$, then the restriction $\omega \mid \sigma$ of $\omega$ to any $m$-simplex $\sigma$ of $M$ is defined and it belongs to the usual Sobolev space $H_{s}^{p}(\sigma)$. In particular, $\omega \mid \sigma$ has weak (and hence strong) derivatives of order $<s$.

(For definitions of weak and strong derivatives see, e.g,. Friedman [F] or Agmon [A].)

Corollary 2.3. If $\omega \in H_{s}^{p}(M)$ and $s>[m / 2]+k$, then $\omega \mid \sigma \in C^{k}(\sigma)$.

Proof of Proposition 2.3. If $\omega \in H_{s}^{p}(M)$, we take $\hat{\Phi}_{s}^{*}(\omega) \in \bigoplus_{\sigma} H_{s}^{p}(\sigma)$ (see (2.12)). The $\sigma$ th component of $\hat{\Phi}_{s}^{*}(\omega)$ will be, by definition, the restriction of $\omega$ to $\sigma$ and it has the desired property.

Corollary 2.3 expresses nothing but the Sobolev theorem (see, e.g., Agmon [A]) in the bounded domain $\sigma$ which has the cone property.

We can prove even global regularity properties for elements of $H_{s}^{p}(M)$.

Propostrion 2.4. (i) If $P(d, \delta)$ is any polynomial in $d, \delta$ and $\omega \in H_{s}^{*}(M)$, $\operatorname{deg} P<s$, then $P(d, \delta) \omega$ is well defined and belongs to $H_{s-\operatorname{deg} P}^{*}(M)$. $* \omega$ is well defined and belongs to $H_{s}^{*}(M)$.

(ii) If $\omega, \vartheta \in H_{s}^{*}(M), s>1$, then

$$
(d \omega, \vartheta)_{0}=(\omega, \delta \vartheta)_{0} .
$$

Proof. (i) Observe that the scalar product $(,)_{s}[(2.1)]$ can be written also as

$$
\begin{aligned}
(\alpha, \beta)_{s} & =\sum_{0<i<s}\left(\begin{array}{c}
s \\
i
\end{array}\right)\left((d \delta)^{i}+(\delta d)^{i} \alpha, \beta\right) \\
& =\sum_{0<i<s}\left(\begin{array}{c}
s \\
i
\end{array}\right)\left[\left(W_{i}(d, \delta) \alpha, W_{i}(d, \delta) \beta\right)+\left(W_{i}(\delta, d) \alpha, W_{i}(\delta, d) \beta\right)\right]
\end{aligned}
$$

where $W_{i}(d, \delta)$ is: (a) the unique word of length $i$ which is constructed with the symbols $d, \delta$, (b) begins with $d$ and (c) which does not contain two consecutive $d$ 's or $\delta$ 's.

From (2.14) we deduce, if $\left\{\omega_{n}\right\}$ is a Cauchy sequence in $\left(\Omega^{*}(M),\|\|_{s}\right)$ which converges to $\omega \in H_{s}^{*}(M)$, then $\left\{W_{i}(d, \delta) \omega_{n}\right\}_{n}$ (resp., $\left.\left\{W_{i}(\delta, d) \omega_{n}\right\}_{n}\right)$, $0<i<p$, is a Cauchy sequence in $\left(\Omega^{*}(M),\|\|_{0}\right)$. Hence it converges to an element in $H_{0}^{*}(M)$ which we agree to denote by $W_{i}(d, \delta)$ (resp., $\left.W_{i}(\delta, d) \omega\right)$. We observe also that for $0 \leqslant i \leqslant s-1,\left\{W_{i}(d, \delta) d \omega_{n}\right\}_{n},\left\{W_{i}(\delta, d) d \omega_{n}\right\}_{n}$, (resp. $\left.\left\{W_{i}(d, \delta) \delta \omega_{n}\right\}_{n},\left\{W_{i}(\delta, d) \delta \omega_{n}\right\}_{n}\right)$, are Cauchy sequences in the \|\|$_{0^{-}}$ norm. (2.14) shows again that $\left\{d \omega_{n}\right\}_{n}$ (resp. $\left\{\delta \omega_{n}\right\}_{n}$ ), is a Cauchy sequence in \|\|$_{s-1}$; hence it converges to an element in $H_{s-1}^{*}(M)$ which we agree to indicate by $d \omega$ (resp. $\delta \omega)$. Therefore $d \omega, \delta \omega \in H_{s-1}^{*}(M)$.

We obtain (i) for any polynomial $P(d, \delta)$ by iterating this result.

If $\left\{\omega_{n}\right\}_{n}$ is a Cauchy sequence in $\Omega^{*}(M)$ which converges in the \|\|$_{s}$-norm to $\omega \in H_{s}^{*}(M)$, then by definition 


$$
\left(\Delta^{r}\left(\omega_{j}-\omega_{i}\right),\left(\omega_{j}-\omega_{i}\right)\right)_{0} \stackrel{i j j}{\rightarrow} 0, \quad 0 \leqslant r<s .
$$

But from (4.4) (iv) and the commutativity of $\Delta$ with * , we deduce that

$$
\left(\Delta^{r}\left(* \omega_{j}-* \omega_{i}\right),\left(* \omega_{j}-* \omega_{i}\right)\right)=\left(\Delta^{r}\left(\omega_{j}-\omega_{i}\right),\left(\omega_{j}-\omega_{i}\right)\right),
$$

which proves $\left\{{ }^{*} \omega_{n}\right\}_{n}$ is still Cauchy in the \|\|$_{s}$-norm.

(ii) It is sufficient to suppose $\omega, \sigma \in H_{1}^{*}(M)$. Let $\left\{\omega_{n}\right\}_{n} \stackrel{\|\|_{1}}{\rightarrow} \omega$ (resp., $\left\{\vartheta_{n}\right\} \stackrel{\|\|_{1}}{\rightarrow} \vartheta$ ), be a sequence in $\Omega^{*}(M)$ which converges in the \|\|$_{1}$-norm to $\omega$ (resp., $\vartheta$ ). Then we have by (i):

We get

$$
\begin{array}{lll}
\omega_{n} \stackrel{\|\|_{0}}{\rightarrow} \omega, & d \omega_{n} \stackrel{\|\|_{0}}{\rightarrow} d \omega, & \delta \omega_{n} \stackrel{\|\|_{0}}{\rightarrow} \delta \omega_{n}, \\
\vartheta_{n} \stackrel{\|\|_{0}}{\rightarrow} \vartheta, & d \delta_{n} \stackrel{\|\|_{0}}{\rightarrow} d \vartheta, & \delta \vartheta_{n} \stackrel{\|\|_{0}}{\rightarrow} \delta \vartheta .
\end{array}
$$

$$
(d \omega, \delta)=\lim _{n}\left(d \omega_{n}, \vartheta_{n}\right)=\lim _{n}\left(\omega_{n}, \delta \vartheta_{n}\right)=(\omega, \delta \vartheta) .
$$

THEOREM 2.5. If $\omega \in H_{s}^{p}(M), s>[m / 2]+k$, then $\omega$ is a $p$-form of class at least $k$ on $M$.

Proof. Corollary 2.3 says that $\omega / \sigma$ is a form of class $C^{k}$ for any $m$-simplex $\sigma \in M$. If $\sigma, \tau$ are two neighboring simplices in $M$, then $\sigma \cup \tau$ can be thought of as a bounded domain in $\mathbf{E}^{n}$ with the cone property, and hence the Sobolev theorem in $\sigma \cup \tau$ says that the form $\{\omega|\sigma, \omega| \tau\}$ on $\sigma \cup \tau$ is of class at least $k$; hence $\sigma \mapsto \omega_{\sigma}$ is a form of class $C^{k}$ on $M$.

Corollary 2.5'. If $\omega \in H_{s}^{p}(M)$ for any $s$, then $\omega \in \Omega^{p}(M)$. Hence $H_{\infty}^{p} \stackrel{\text { def }}{=}$ $\cap_{s} H_{s}^{p}$ is $\Omega^{p}(M)$.

3. Sobolev spaces and distributions. As in the case of the classical distributions, and the classical Sobolev spaces, any element $u \in H_{s}^{*}, s \in \mathbf{Z}$, defines a distribution. Really, if $s>0$, then $u \in H_{0}^{*}$ and we can associate with $u$ a distribution $T_{u}$, so

$$
T_{u}(\varphi)=\int_{M} u \wedge \varphi=(u, * \varphi), \quad \varphi \in \Omega^{*}(M) .
$$

Any element $u \in H_{-s}, s>0$, is by definition a continuous functional on $H_{s}^{*}$; that means there exists a unique element $u_{s} \in H_{s}^{*}$ such that

$$
u(\varphi)=\left(u_{s}, * \varphi\right)_{s}=\left(u_{s},(1+\Delta)^{s} * \varphi\right)_{0}, \quad \text { for any } \varphi \in \Omega *(M) ;
$$

$u$ is clearly a distribution. The uniqueness of $u_{s}$ permits us to formulate:

Proposition 3.1. The correspondence

$$
\begin{aligned}
& x: H_{-s}^{*}(M) \rightarrow \mathscr{D} *(M), \quad s>0, \\
& u \mapsto u(), \\
& u(\varphi)=\left(u_{s},(1+\Delta)^{s} * \varphi\right)_{0}, \quad \varphi \in \Omega^{*}(M), \quad u_{s} \in H_{s}^{*}(M),
\end{aligned}
$$

is injective. 
Any element in $\chi\left(H_{-s}^{*}(M)\right)$ will be called distribution in $H_{-s}^{*}(M), s>0$.

Proposition 3.2. $\chi H_{-s}^{*}(M)=\left\{u \mid u \in \mathscr{D} *(M), u(\varphi)=\Sigma\left(a_{i}, P_{i}(d, \delta) * \varphi\right)_{0}\right.$, $\varphi \in \Omega^{*}(M), a_{i} \in H_{0}^{*}(M), P_{i}$ polynomials in $d, \delta$ of degree $\left.<s\right\}$.

Proof. Proposition 3.1 and Proposition 2.4 give us that any distribution $u$ in $H_{-s}^{*}(M)$ is of the form

$$
\varphi \mapsto \sum_{i}\left(a_{i}, P_{i}(d, \delta) * \varphi\right)_{0}, \quad \varphi \in \Omega *(M) .
$$

Conversely, any functional (3.3) is a distribution. This functional is continuous in the \|\|$_{s}$-norm because for any polynomial $P_{i}$ there exists a constant $C_{i}>0$, such that

$$
\left\|P_{i}(d, \delta) * \varphi\right\|_{0}^{2} \leqslant C_{i}\left(\varphi,(1+\Delta)^{\operatorname{deg} P_{i}} \varphi\right)=C_{i}\|\varphi\|_{\operatorname{deg} P_{i}}^{2}<C_{i}\|\varphi\|_{s}^{2}
$$

hence

$$
\left|\sum_{i}\left(a_{i}, P_{i}(d, \delta) * \varphi\right)_{0}\right|<\left|\sum_{i}\left(a_{i}, P_{i}(d, \delta) * \varphi\right)_{0}\right|<\sum_{i} C_{i}\left\|a_{i}\right\|_{0} \cdot\|\varphi\|_{s}
$$

Therefore the distribution (3.3) stays in $H_{-s}^{*}(M)$.

4. Cohomology. The results of this section remain valid even for homological PL-manifolds (for definition, see Milnor [Mi]).

Definition 4.1. Let $A \subset U \subset M$ be open sets. Let us define

$$
\begin{aligned}
\Omega^{p}(U, A) & =\left\{\omega \mid \text { there exists } \tilde{\omega} \in \Omega^{p}(M) \text { such that } \tilde{\omega}|U=\omega, \omega| A=0\right\}, \\
\mathscr{D}^{p}(U, A) & =\left\{T \mid \text { there exists } \tilde{T} \in \mathscr{D}^{p}(M) \text { such that } \tilde{T}|U=T, T| A=0\right\}, \\
\Omega^{p}(U) & =\Omega^{p}(U, \Phi), \\
\mathscr{D}^{p}(U) & =\mathscr{D}^{p}(U, \Phi), \\
\Omega_{0}^{p}(U) & =\left\{\omega \mid \omega \in \Omega^{p}(U), \text { Supp } \omega \subset U\right\}, \\
\mathscr{D}_{0}^{p}(U) & =\left\{T \mid T \in \mathscr{D}^{p}(U), \text { Supp } T \subset U\right\} .
\end{aligned}
$$

It is clear that $\left\{\Omega^{p}(U, A), d\right\}=\Omega^{*}(U, A)$ and $\left\{\mathscr{D}^{p}(U, A), d\right\}=\mathscr{D} *(U, A)$ are cochain complexes and let us denote their homologies by

$$
\begin{aligned}
H_{p}\left(\Omega^{*}(U, A)\right) & =H^{p}(U, A), & p>0, \\
H_{p}(\mathscr{Q} *(U, A)) & =\hat{H}^{p}(U, A), & p>0 .
\end{aligned}
$$

THEOREM 4.1. If $M$ is any combinatorial manifold, then the inclusion

induces the isomorphism

$$
j: \Omega^{*}(M) \rightarrow \mathscr{D} *(M)
$$

$$
j_{*}: H^{*}(M) \rightarrow \hat{H}^{*}(M)
$$

in cohomology. 
The inclusion of the complex $\Omega^{*}(M)$ in the complex of PL-differential forms induces isomorphism in cohomology.

Therefore all these cohomologies are isomorphic with $H^{*}(M, \mathrm{R})$.

Proof of Theorem 4.1. We shall prove the lemmas:

LEMMA 4.1. The sheaves $\Omega^{*}(M), \underline{\mathscr{D} *(M)}$ are fine.

LEMMA 4.2. The diagram

$$
\begin{aligned}
& 0 \rightarrow \underset{\|}{\mathbf{R}} \rightarrow \frac{\Omega^{0}(M)}{\downarrow j_{0}} \stackrel{d}{\rightarrow} \frac{\Omega^{1}(M)}{\downarrow j_{1}} \stackrel{d}{\rightarrow} \ldots \stackrel{d}{\rightarrow} \frac{\Omega^{m}(M)}{\downarrow j_{m}} \rightarrow 0 \\
& 0 \rightarrow \mathbf{R} \rightarrow \underline{\mathscr{D}^{0}(M)} \stackrel{d}{\rightarrow} \underline{\mathscr{D}^{1}(M)} \stackrel{d}{\rightarrow} \ldots \stackrel{d}{\rightarrow} \underline{\mathscr{D}^{m}(M)} \rightarrow 0
\end{aligned}
$$

is commutative.

LEMMA 4.3 ("POINCARE" LEMMA). The rows in (4.3) are exact.

Theorem 4.1 will follow from the generalized de Rham theorem.

Lemma 4.1 will be proved in Appendix I.

Lemma 4.2 is evident.

Proof OF Lemma 4.3. Let $x_{0}$ be any point in $M$. If $x_{0}$ is an internal point of a maximal simplex, then exactness of (4.3) in $x_{0}$ is a particular case of the de Rham isomorphism theorem (see de Rham [R]). Therefore it remains to prove exactness of (4.3) in any point $x_{0}$ on the boundary of a maximal simplex in $M$. So, suppose $x_{0}$ is an internal point of a simplex $\tau \in M, 0<\operatorname{dim} \tau=k<$ $m$.

An open neighborhood $U$ of $x_{0}$ in $M^{m}$ will be called "normal" iff $U$ is combinatorially and metrically equivalent to a product $I^{k} \times B^{m-k}$ where $I^{k}$ is a $k$-cube in $\tau$, containing $x_{0}$, and $B^{m-k}$ is a combinatorial disc of dimension $m-k ; B^{m-k}$ can be chosen to be a combinatorial disc in $M$, containing $x_{0}$ and "normal" to $\tau$ (with respect to the Riemannian metric $\Gamma$ ).

We need the following:

SUBLEMMA 4.4. If $U$ is any normal neighborhood of $x_{0} \in \tau \subset M$, then

(i) $H^{i}(U) \simeq H^{i}\left(\left\{x_{0}\right\}, \mathbf{R}\right), \quad i>0$,

(ii) $\hat{H}^{i}(U) \cong H^{i}\left(\left\{x_{0}\right\}, \mathbf{R}\right), \quad i>0$.

Proof of Sublemma 4.4. The proof of (i) follows easily by using the classical homotopy operator $h$ (see, e.g., de Rham [RD). Let $\sigma \in M$ be any simplex which contains $x_{0}$, and let $x_{\sigma}$ be the chosen coordinate system on $\sigma$. Let $\bar{x}_{\sigma}$ be the coordinate system obtained from $x_{\sigma}$ by translating the origin in $x_{0}$. Let $\sigma \mapsto \omega_{\sigma}$ be any form $\omega$ in $\Omega^{p}(U)$. Then $h \omega$ is a form defined locally, whose restriction to $\sigma$ is 


$$
\left(h \omega_{\alpha}\right)(\bar{x})=\left\{\begin{array}{l}
\omega_{\alpha}\left(\bar{x}_{0}\right), \quad \text { for } p=0, \\
\sum_{k=1}^{k=p}(-1)^{k-1} \int_{0}^{1} t^{p-1} \bar{x}^{i_{k}} \omega_{i_{1}} \cdots j_{p}(\bar{t} \bar{x}) d t \\
\wedge d \bar{x}^{i_{1}} \wedge \cdots \wedge \widehat{d \bar{x}}^{i_{k}} \wedge \cdots \wedge d \bar{x}^{i}, \quad p>1,
\end{array}\right.
$$

where

$$
\omega=\omega_{i_{1} \ldots i_{p}} d \bar{x}^{i_{1}} \wedge \cdots \wedge d \bar{x}^{i_{j}}
$$

The formula (4.5) is clearly invariant under affine transformations, hence $h \omega$ satisfies the $C^{\infty}$-compatibility conditions. Therefore $h \omega \in \Omega^{p-1}\left(U\left(x_{0}, r\right)\right)$.

The operator $h$ satisfies the identities

$$
\begin{aligned}
& \omega(x)-\omega(0)=h d \omega, \text { for } \omega \in \Omega^{0}(U), \\
& (h d+d h) \omega=\omega, \text { for } \omega \in \Omega^{p}(U), p>1 .
\end{aligned}
$$

The relations (4.6) imply 4.4(i).

The proof of 4.4(ii) is not straightforward. We prove it by induction on $\operatorname{dim} M$.

For $m=1,4.4$ (ii) reduces at the smooth case (for its proof see de Rham [R] or Schwartz [Sc]).

SUBLEMMa 4.5. Let $U_{0}, U$ be two open normal neighborhoods of $x_{0} \in M$ ( $U_{0} \cap \tau, U \cap \tau$ are homothetic cubes) and $\bar{U}_{0} \subset U$. Let us denote $V=U-$ $\bar{U}_{0}$. Then

$$
\hat{H}^{i}(V) \simeq H^{i}\left(\mathbf{S}^{m-1}, \mathbf{R}\right), \quad i>0
$$

Proof of Sublemma 4.5 will be given at the end of this section.

We have the exact sequence of complexes

$$
0 \rightarrow \mathscr{D} *(U, V) \stackrel{\iota}{\rightarrow} \mathscr{D} *(U) \stackrel{\pi}{\rightarrow} \mathscr{D} *(V) \rightarrow 0
$$

where $\iota$ is inclusion while $\pi$ is given by restriction of distributions to $V$.

The exact sequence (4.8) gives a long exact sequence in cohomology:

$\ldots \rightarrow \hat{H}^{p-1}(V) \rightarrow \hat{H}^{p}(U, V) \stackrel{\text { p }}{\rightarrow} \hat{H}^{p}(U) \stackrel{\pi_{p}}{\rightarrow} \hat{H}^{p}(V) \stackrel{\delta_{p}}{\rightarrow} \hat{H}^{p+1}(U, V) \rightarrow \ldots$

We deduce from Sublemma 4.5 and (4.9)

$$
\hat{H}^{p}(U, V) \stackrel{\text { s }}{\rightarrow} \hat{H}^{p}(U) \text { for } 1<p<m-2,
$$

is an isomorphism; the last terms in (4.10) are

$$
0 \rightarrow \hat{H}^{m-1}(U, V) \stackrel{m_{m-1}}{\rightarrow} \hat{H}^{m-1}(U) \stackrel{\pi_{m-1}}{\rightarrow} \mathbf{R} \stackrel{\delta_{m-1}}{\rightarrow} \hat{H}^{m}(U, V) \stackrel{m_{m}}{\rightarrow} \hat{H}^{m}(U) \stackrel{\pi_{m}}{\rightarrow} 0
$$

Let $T$ be any distribution in $\mathscr{D}^{p}(U, V), 0<p<m$, and let us consider the 
distribution $H T$ defined as

$$
(H T)(\omega)=T(h \omega) \text { for any } \omega \in \Omega_{0}^{*}(U) .
$$

$T$ being with compact support in $U, H T$ is well defined (observe that $h \omega$ is not necessarily with support in $U$ ). $H$ satisfies the identity

$$
d H T+H d T=T \text { for any } T \in \mathscr{T}^{p}(U, V), \quad 0<p<m-1 .
$$

Suppose $d T=0$; then (4.13) yields $T=d H T$. Observe that $H T$ does not stay necessarily in $\mathscr{Q}^{p-1}(U, V)$ because the support of $h \omega$ (for any $\omega \in$ $\left.\Omega_{0}^{p^{-1}}(V)\right)$ is not necessarily in $V$. In any case, $H T$ belongs to $\mathscr{Q}^{p-1}(U)$ and hence, here, $H T$ is a coboundary. Therefore, if $[T]$ denotes the cohomology class of $T$ in $\hat{H}^{p}(U, V)$, we have

$$
\iota_{p}[T]=0 \in \hat{H}^{p}(U) \text {. }
$$

But, as $l_{p}, 1<p \leqslant m-2$, is an isomorphism, we deduce

$$
\hat{H}^{p}(U, V)=\hat{H}^{p}(U)=0, \quad 1<p<m-2 .
$$

(4.11) shows that $\iota_{m-1}$ is a monomorphism, hence the same argument can be used in order to prove also that

$$
\hat{H}^{m-1}(U, V)=0 .
$$

Let $T$ be any cocycle in $\mathscr{D}^{m}(U)$. From (4.11) we get that $\iota_{m}$ is an epimorphism. Hence there exists a cocycle $T_{1} \in \mathscr{Q}^{m}(U, V)$ which stays in the cohomology class of $T . T_{1}$ having compact support in $U$, it makes sense to compute $T_{1}(\omega)$ for any $\omega \in \Omega^{0}(U)$; we have by (4.6)

$$
\begin{aligned}
T_{1}(\omega) & =T_{1}\left(\omega\left(x_{0}\right)+h d \omega\right)=T_{1}\left(\omega\left(x_{0}\right)\right)+H T_{1}(d \omega) \\
& =T_{1}\left(\omega\left(x_{0}\right)\right)+d H T_{1}(\omega) .
\end{aligned}
$$

Let $\delta_{x_{0}} \in \mathscr{Q}^{m}(U)$ denote the Dirac distribution, i.e.,

$$
\delta_{x_{0}}(\omega)=\omega\left(x_{0}\right) \text { for any } \omega \in \Omega^{0}(U) .
$$

Therefore, from (4.17) we get

$$
[T]=\left[T_{1}\right]=a\left[\delta_{x_{0}}\right] \in \hat{H}^{m}(U),
$$

where $a=T_{1}(1)$.

We shall prove that

$$
\delta_{0}=d G, \quad G \in \mathscr{P}^{m-1}(U) .
$$

Let us define

$$
G(\omega)=\frac{-1}{|\partial U|} \int_{\partial U} h_{1} \omega d s \quad \text { for any } \omega \in \Omega_{0}^{1}(U) \text {, }
$$

where $d s$ denotes the Riemann measure on $\partial U$ induced by the flat metric $\Gamma$ on $M$, and $|\partial U|$ denotes the measure of $\partial U$. 
We have, for any $\omega \in \Omega_{0}^{0}(U)$,

$$
\begin{aligned}
(d G) \omega & =G(d \omega)=\frac{-1}{|\partial U|} \int_{\partial U} h_{1} d \omega d s=\frac{-1}{|\partial U|} \int_{\partial U}\left(\omega-\omega\left(x_{0}\right)\right) d s \\
& =\omega(0) \frac{1}{\mid \partial U} \int_{\partial U} d s=\delta_{x_{0}}(\omega)
\end{aligned}
$$

(observe that $\omega \mid \partial U \equiv 0$ ). Therefore

$$
\hat{H}^{m}(U)=0 .
$$

At this point we know from (4.16) and (4.21) that (4.11) reduces to the exact sequence

$$
0 \rightarrow \hat{H}^{m-1}(U) \rightarrow \mathbf{R} \rightarrow \hat{H}^{m}(U, V) \rightarrow 0 .
$$

We shall prove that $\operatorname{dim} \hat{H}^{m}(U, V)>1$; then (4.22) will imply that

$$
\hat{H}^{m-1}(U)=0, \quad \hat{H}^{m}(U, V) \simeq \mathbf{R} .
$$

The Dirac distribution $\delta_{x_{0}}$ is a cocycle in $\mathscr{D}^{m}(U, V)$. We shall prove that its cohomology class in $\hat{H}^{m}(U, V)$ is not the null class. Suppose the contrary, say $\delta_{x_{0}}=d S, S \in \mathscr{Q}^{m-1}(U, V)$. Then for $1 \in \Omega^{0}(U)$ we obtain the contradiction

$$
1=\delta_{x_{0}}(1)=d S(1)=S(d 1)=S(0)=0 .
$$

Therefore (4.23) is true.

Let us compute $\hat{H}^{0}(U)=\operatorname{Ker} d_{0}$. Let $T \in \operatorname{Ker} d_{0}$. Then

$$
0=(d T)(\omega)=T(d \omega) \text { for any } \omega \in \Omega_{0}^{m-1}(U) .
$$

We observe that

$$
\Omega_{0}^{m}(U) / d \Omega_{0}^{m-1}(U) \simeq H^{m}\left(S^{m}, \mathbf{R}\right) \approx \mathbf{R} .
$$

Therefore $\operatorname{dim} \hat{H}^{0}(U)<1$.

Let $f \in \Omega^{0}(U)$ be any constant function. Then $f$ thought of as a distribution is a cocycle. For, let $T_{f}$ denote the distribution associated with $f$, i.e.,

$$
T_{f}(\omega)=\int_{U} f \omega=f \int_{U} \omega \text { for any } \omega \in \Omega_{0}^{m}(U)
$$

Then we have

$$
d T_{f}(\omega)=f \int_{U} d \omega=0
$$

Therefore

$$
\hat{H}^{0}(U)=H^{0}(U) \simeq \mathbf{R}
$$

Proof of Sublemma 4.5. If we show that the sequence of sheaves and morphisms

$$
0 \rightarrow \mathbf{R} \rightarrow \mathscr{D}^{0}(V) \stackrel{d}{\rightarrow} \mathscr{Q}^{1}(V) \stackrel{d}{\rightarrow} \cdots \rightarrow \underline{\mathscr{Q}^{m}(V)} \rightarrow 0
$$

is exact, then the Sublemma 4.5 is proved. 
We emphasize that exactness of (4.27) is simpler to show than exactness of (4.3). This is produced by the fact that for any $x_{0} \in V$, any normal neighborhood is of the form

$$
U=\tilde{U} \times(-r, r)
$$

where $\tilde{U}$ is a normal neighborhood of $x_{0}$ in a combinatorial manifold of dimension $m-1$. Really, if $x_{0}$ is an interior point of a maximal simplex, it is clearly true. If $x_{0}$ is a point on the boundary of a maximal simplex $\sigma$ of the decomposition $M$, observe that in $V$ any vertex of the decomposition $M$ is avoided, and then any normal neighborhood of $x_{0}$ is of the form (4.28).

At this point we use an induction argument on $\operatorname{dim} M$. Suppose we have proved the Poincaré Lemma 4.3, for all $M$ of dimension $m-1$. Hence we may suppose that the sequence of sheaves and homomorphisms

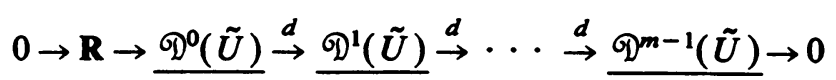

is exact, and we shall prove that (4.27) is exact. In order to perform this, we use Schwartz's proof [Sc, Chapter IX] for the Poincare lemma.

The basic tool used by Schwartz is convolution by the Heaviside distribution in order to construct primitives.

We shall show that the linear factor $(-r, r)$ in (4.28) is sufficient for constructing "partial convolutions" and then primitives.

Let us consider $\tilde{U} \times \mathbf{R} \supset \tilde{U} \times(-r, r)$, and let $(y, t)$ be a generic point in $\tilde{U} \times \mathbf{R}$.

LEMMA 4.6. Let $T$ be a distributional form on $\tilde{U} \times \mathbf{R}$ and $S$ any distributional form on $\mathbf{R}$.

(i) Then it is possible to define a distributional form $T \otimes S$ on $\tilde{U} \times \mathbf{R} \times \mathbf{R}$, such that, when $U$ is an open set in $\mathbf{R}^{m-1}$, then $T \otimes S$ is the usual tensor product of the distributional forms $T, S$.

(ii) Let $\mu: \tilde{U} \times \mathbf{R} \times \mathbf{R} \rightarrow \tilde{U} \times \mathbf{R}$ be the map

$$
\mu(y, t, s)=(y, t+s) .
$$

Suppose $T$ has compact support. Then it makes sense to define "partial convolution product" $T * S$ on $\tilde{U} \times \mathbf{R}$ as follows

$$
(T * S)(\omega)=(T \otimes S)\left(\mu^{*} \omega\right), \text { for any } \omega \in \Omega_{0}^{*}(\tilde{U} \times \mathbf{R}) .
$$

(iii) The partial convolution product satisfies

$$
\frac{\partial}{\partial t}(T * S)=T * \frac{\partial S}{\partial t}
$$

(For a sketch of the proof, see Appendix II.)

From now on, the exactness of (4.27) can be carried out as in Schwartz [Sc, Chapter IX]. 
5. Combinatorial Hodge theory. For any $s \in Z$, let us define spaces of harmonic forms

$$
\mathcal{H}_{s}^{P}(M)=\left\{\omega \mid \omega \in H_{s}^{P}(M), d \omega=0=\delta \omega\right\}
$$

TheOREM 5.1 (COMBINATORIAL Hodge DeCOMPOSITION THEOREM). Let $s$ be any integer $>1$. Then:

(i) $\mathcal{H}_{s}(M)$ is finite dimensional for any $0<p<m$,

(ii) for any $f \in H_{s-1}^{p}(M), 0<p<m$, there exist a unique $h \in \mathcal{S}_{s}^{p}(M)$ and a unique $u\left(\bmod \mathcal{F}_{s}^{P}(M)\right)$ in $H_{s}^{P}(M)$ such that

$$
(1+\Delta)^{s-1} f=(1+\Delta)^{s-1}(h+\Delta u)=h+(1+\Delta)^{s-1} \Delta u
$$

in the distributional sense.

Proof. The proof is substantially the same as in the case of the Dirichlet problem.

Let $B_{s}(u, \phi)$ be the bilinear form defined on $H_{s}^{*}(M), s>1$,

$$
B_{s}(u, \phi)=(d u, d \phi)_{s-1}+(\delta u, \delta \phi)_{s-1}, \quad u, \phi \in H_{s}^{*}(M) .
$$

We have the "Gårding-equality":

$$
B_{s}(u, \phi)+(u, \phi)_{s-1}=(u, \phi)_{s} \text { for any } u, \phi \in H_{s}^{*}(M) \text {. }
$$

We intend to determine the elements $u \in H_{s}^{*}(M)$ which satisfy the equation

$$
B_{s}(u, \phi)=(f, \phi)_{s-1} \text { for any } \phi \in H_{s}^{*}(M)
$$

where $f$ is a given element in $H_{s-1}^{*}(M)$.

This last problem and the Dirichlet problem are expressed by the same functional analysis problem. For its solution, see, e.g., Agmon [A, Theorem 8.5, p. 102].

It follows that the space of solutions in $H_{s}^{*}(M)$ of the homogeneous equation $B_{s}(u, \varphi)=0$ is finite dimensional and the equation (5.3) has solution iff $f$ is orthogonal (in the \|\|$_{s-1}$-norm) to the space of solutions of the homogeneous equation (5.3). Notice that the space of solutions in $H_{s}^{*}(M)$ of the homogeneous equation (5.3) is just $\mathcal{K}_{s}^{*}(M)$. Indeed, if $u$ is such a solution, let us put $\phi=u$ in (5.3); we have

$$
(d u, d u)_{s-1}+(\delta u, \delta u)_{s-1}=0,
$$

and hence $d u=0=\delta u$.

Suppose now $u$ is a solution of the nonhomogeneous equation (5.3).

Take $\phi \in \Omega^{*}(M)$ in (5.3) and remember (5.2). We get

$$
(d u, d \phi)_{s-1}+(\delta u, \delta u)_{s-1}=(f, \phi)_{s-1}
$$

or, as $s>1$,

$$
(u, \delta d \phi)_{s-1}+(u, d \delta \phi)_{s-1}=(f, \phi)_{s-1}
$$


which gives us

$$
(u, \Delta \phi)_{s-1}=(f, \phi)_{s-1} \text {. }
$$

Let $f$ be any element in $H_{s-1}^{*}(M)$. Since $\mathscr{H}_{s}^{*}(M)$ is finite dimensional, it is closed in $H_{s-1}^{*}(M)$. Let $h$ denote the projection (with respect to $\left.(,)_{s-1}\right)$, of $f$ on $\mathcal{C}_{s}^{*}(M)$. Then $f-h$ is orthogonal to $\mathscr{T}_{s}^{*}(M)$; hence the equation (5.3), or

$$
(1+\Delta)^{s-1} f=(1+\Delta)^{s-1}(h+\Delta u)
$$

has a solution. Theorem 5.1 is completely proved.

Any harmonic form $h \in \mathcal{T}_{s}^{p}(M)$ is, in particular, a $d$-cocycle in $\mathscr{Q}^{p}(M)$. Let $[h]$ denote its cohomology class in $\hat{H}^{p}(M)$.

The map

$$
\begin{aligned}
\chi_{s}^{p}: \mathcal{T}_{s}^{p}(M) \rightarrow \mathscr{P}^{p}(M), \quad & 0<p<m,-\infty<s<+\infty, \\
h & \mapsto[h]
\end{aligned}
$$

will be called "Hodge homomorphism".

THEOREM 5.2. (i) The Hodge homomorphism

$$
\chi_{1}^{p}: \mathscr{T}_{1}^{p}(M) \rightarrow \hat{H}^{p}(M), \quad 0<p<m,
$$

is a monomorphism.

(ii) The Hodge homomorphism

$$
\chi_{-1}^{p}: \mathcal{F C}_{-1}^{p}(M) \rightarrow H^{p}(M), \quad 0<p<m,
$$

is an epimorphism.

Proof of Theorem 5.2(i). The idea of the proof is to show that if $h \in \mathcal{H} \mathcal{C}_{1}^{*}(M)$, and the cohomology class of $h$ is zero, then there exists a sufficiently regular form $u$ such that: $d u=h$.

At this point we may proceed as in the classical case. Let $T$ denote any triangulation which is a refinement of the initial decomposition of $M$. Let us define a new space of distributions $W_{1}^{*}(M, T) \subseteq H_{0}^{*}(M)$ which contains $H_{1}^{*}(M)$.

Definition 5.1. Let us define, for any $0<r<\operatorname{dim} M$, $W_{1}^{r}(M, T)=\left\{\omega \mid \omega \in H_{0}^{r}(M)\right.$ such that: (i) for any maximal simplex

$$
\begin{aligned}
& \sigma_{\alpha} \in T, \omega \mid \sigma_{\alpha} \in H_{1}^{*}\left(\sigma_{\alpha}, \Lambda^{r} T^{*} \sigma_{\alpha}\right), \\
& \text { (ii) for any two maximal neighboring } \\
& \text { simplices } \sigma_{\alpha}, \sigma_{\beta} \text {, the equality } \\
& \left.\qquad \omega\left|\sigma_{\alpha}=\omega\right| \sigma_{\beta} \text { holds in } H_{0}^{r}\left(\sigma_{\alpha} \cap \sigma_{\beta}\right)\right\} .
\end{aligned}
$$

Explanation. $\omega_{\gamma} \mid \sigma_{\alpha} \cap \sigma_{\beta}(\gamma=\alpha, \beta)$ is the restriction of the form $\omega$ to the submanifold $\sigma_{\alpha} \cap \sigma_{\beta}$ of codimension 1; it belongs to $H_{0}^{r}\left(\sigma_{\alpha} \cap \sigma_{\beta}\right)$, as a particular case of the Sobolev trace theorem. 
DEFINITION 5.2. Let

$$
\hat{W}_{1}^{r}(M, T)=\left\{\omega \mid \omega \in W_{1}^{r}(M, T), d \omega \in W_{1}^{r+1}(M, T)\right\},
$$

$d$ denoting strong exterior derivative.

Clearly, $\left\{\hat{W}_{1}^{r}(M, T), d\right\}_{r}$ is a complex, and let us denote it by $\hat{W}_{1}^{*}(M, T)$.

LEMMA 5.1. (i) The triangulation $T$ being finer than the fixed decomposition of $M$, the inclusion

$$
H_{1}^{r}(M) \subseteq W_{1}^{r}(M, T)
$$

holds,

(ii) if the triangulation $T$ is sufficiently fine, then the inclusion

$$
\Omega^{*}(M) \stackrel{i}{\rightarrow} \hat{W}_{1}^{*}(M, T) \stackrel{j}{\rightarrow} H_{0}^{*}(M)
$$

induces isomorphisms in cohomology,

(iii) if $u \in W_{1}^{*}(M, T)$ and $h \in H_{1}^{*}(M)$, then

$$
(h, d u)=(\delta h, u)
$$

Suppose for a while Lemma 5.1 has been proved, and let us end the proof of Theorem 5.2(i).

Let $h \in \mathcal{G}_{1}(M)$ and suppose $\chi_{i}^{r}(h)=0$. By Lemma 5.1(ii), there exists a $u \in W_{1}^{r-1}(M, T)$ such that

$$
h=d u \text {. }
$$

We have by (5.16), (5.15)

$$
\|h\|_{0}^{2}=(h, h)=(h, d u)=(\delta h, u)=(0, u)=0 ;
$$

hence $h=0$. Therefore $\chi_{1}^{r}$ is a monomorphism.

Proof of Lemma 5.1.(i) If $\omega \in H_{1}^{*}(M)$, then Proposition 2.3 says that, for any maximal simplex $\sigma_{\alpha}$ of $T$, the restriction $\omega \mid \sigma_{\alpha}$ belongs to $H_{1}^{*}\left(\sigma_{\alpha}\right)$; hence (i) of Definition 5.1 is verified.

Condition (ii) of Definition 5.1 follows immediately because the elements in $H_{1}^{*}(M)$ are limits of elements of class $C^{\infty}$. Therefore $\omega \in W_{1}^{*}(M, T)$.

(ii) Let $\hat{W}_{1}^{r}(M, T)$ denote the sheaf of germs of forms in $\hat{W}_{1}^{r}(M, T)$. This sheaf is fine; if we prove that the sequence

$$
0 \rightarrow \mathbf{R} \rightarrow \underline{W_{1}^{0}(M, T)} \rightarrow \cdots \stackrel{d}{\rightarrow} \underline{W_{1}^{r}(M, T)} \stackrel{d}{\rightarrow} \underline{W_{1}^{r+1}(M, T)} \rightarrow \ldots
$$

is exact, then the generalized de Rham theorem would complete the proof of (ii).

We shall use Hörmander's [H] method of densities in order to prove exactness of (5.18).

Let us check the exactness of (5.18) at the point $x_{0} \in M$. We suppose that the triangulation $T$ is sufficiently fine so that the interior of $\operatorname{Star}\left(x_{0}, T\right)$ is 
simplicially equivalent to a subcomplex of $\mathbf{R}^{m}$ (otherwise we refine it). So we may suppose that $T$ satisfies this property for any point $x_{0} \in M$. Let

be such a simplicial mapping.

$$
\rho: \text { Int } \operatorname{Star}\left(x_{0}, T\right) \rightarrow \mathbf{R}^{m}
$$

Let $f \in \hat{W}_{1}^{r}(M, T)_{x_{0}}$ be such that $d f=0$ (here $d$ denotes strong exterior derivative). Let us suppose, for simplicity, that $f$ is defined on all $\operatorname{Star}\left(x_{0}, M\right)$.

Let $f$ denote the transfer of $f$ by $\rho^{-1}$ in $\mathbf{R}^{m}$, i.e., $\bar{f}=\left(\rho^{-1}\right)^{*} f$.

Let $\Omega=\rho\left(\operatorname{Int} \operatorname{Star}\left(x_{0}, T\right)\right)$.

Observe that $f \in \hat{W}_{1}^{r}\left(\Omega, T^{\prime}\right)$ for a suitable triangulation $T^{\prime}$ of $\Omega$. Observe also that $d f=0$ in the strong sense.

SUBLEMMA 5.2. If $f \in \hat{W}_{1}^{r}\left(\Omega, T^{\prime}\right)$ and $d f=0$ in the strong sense, then $d f=0$ in the weak sense (with respect to all classical $C^{\infty}$-forms on $\Omega$ ).

We shall postpone the proof of Sublemma 5.2.

LemMa 5.3 (Hörmander [H, TheOREM 4.2.5]). Let $\Omega$ be a pseudoconvex open set in $\mathbf{C}^{m}$ and let $0<s$. Then the equation $\bar{\partial} u=f$ has a solution in $W_{s+1}^{p, q}(\Omega, 10 c)$ for every $f \in W_{s}^{p, q+1}(\Omega$, loc) such that $\bar{\partial} f=0$.

Hörmander's theorem remains valid (with the same proof) if we substitute $\mathbf{C}^{m}$ by $\mathbf{R}^{m}, \bar{\partial}$ by $d$, and $W_{s}^{p, q}(\Omega, l o c)$ by $H_{s}^{P}(\Omega, l o c) . f$ belongs to $H_{0}^{r}(\Omega)$ and $d f=0$ in the weak sense. Therefore, there exists a $u \in H_{1}^{r}(\Omega, l o c)$ such that $d u=f$ in the weak sense, and hence $d u=f$ in the strong sense. Then the germ at $x_{0}$ of $\rho^{*} u$ belongs to $\hat{W}_{1}^{r-1}(M, T)_{x_{0}}$ and $d u=f$ in the strong sense, which proves the exactness of (5.18).

Proof of Subleman 5.2. Let $\rho$ be any $C^{\infty}$-form on $\Omega$, Supp $\rho \ll \Omega$. Let the $\sigma_{\alpha}$ denote all maximal simplices of the decomposition $T$.

We have

$$
\begin{aligned}
\int_{\Omega} d \rho & \wedge \bar{f}=\sum_{\alpha} \int_{\sigma_{\alpha}} d \rho \wedge \bar{f}=\sum_{\alpha} \int_{\sigma_{\alpha}}\left(d(\rho \wedge \bar{f})-(-1)^{\operatorname{deg} \rho} \rho \wedge d \bar{f}\right) \\
= & \sum_{\alpha} \int_{\partial \sigma_{\alpha}} \rho \wedge \bar{f}-(-1)^{\operatorname{deg} \rho} \sum_{\alpha} \int_{\sigma_{\alpha}} \rho \wedge 0=\sum_{\alpha} \int_{\partial \sigma_{\alpha}} \rho \wedge \bar{f}=0
\end{aligned}
$$

hence $d f=0$ in the weak sense.

Proof of LemMa 5.1 (iii). We have

$$
\begin{aligned}
(h, d u) & =\sum_{\alpha} \int_{\sigma_{\alpha}} d u \wedge * h \\
& =\sum_{\alpha} \int_{\sigma_{\alpha}}\left(d(u \wedge * h)-(-1)^{\operatorname{deg} u} u \wedge d * h\right) \\
& =\sum_{\alpha} \int_{\partial \sigma_{\alpha}} u \wedge * h-(-1)^{\operatorname{deg} u} \sum_{\alpha} \int_{\sigma_{\alpha}} u \wedge d * h \\
& =(-1)^{\operatorname{deg} u} \sum_{\alpha} \int_{\sigma_{\alpha}} u \wedge d * h=(u, \delta h) .
\end{aligned}
$$


Notice that it is important for $h$ to belong to $H_{1}^{*}(M)$; indeed in (5.21) we have used the fact that $* h \in \hat{W}_{1}^{*}(M, T)$, which implied the relation

$$
\sum_{\alpha} \int_{\partial \sigma_{\alpha}} u \wedge * h=0
$$

REMARK 5.1. The spaces

$$
\lim _{T} W_{1}^{r}(M, T), \quad 0<r<m,
$$

are combinatorial invariants.

ProOF OF THEOREM 5.2.(ii) Let $\gamma$ be any cohomology class in $\hat{H}^{P}(M)$. Theorem 4.1 allows us to find a $d$-cocycle $\omega \in \Omega^{P}(M)$ with $[\omega]=\gamma$. In particular, $\omega$ stays in $H_{1}^{P}(M)$. By Theorem 5.1 there exists an element $u \in H_{1}^{P}(M)$ such that

$$
\omega=H \omega+\Delta u,
$$

where $H \omega$ is the orthogonal projection of $\omega$ on the space of harmonic forms $\mathcal{T C}_{1}(M)$. The formula (5.22) can also be written in the sense of distributions

$$
\omega=H \omega+d \delta \omega+\delta d \omega .
$$

Let us apply $d$ to both sides of (5.23); we get $0=d \omega=d \delta d \omega$.

As $\delta \delta d \omega=0$, by Proposition 3.2 we deduce that $\delta d \omega \in \mathcal{I C P}(M)$.

Therefore both $H \omega+\delta d \omega$ and $\omega$ stay in the same cohomology class in $\hat{H}^{P}(M)$; Theorem 5.2 is completely proved.

6. Combinatorial analogue of the signature operator. In analogy with the signature operator defined on smooth Riemannian manifolds, let us define the operator $D=d+\delta$.

Proposimon 6.1. If $u \in H_{1}^{*}$, and $D u=0$, then $d u=0, \delta u=0$.

Proof. We have for any $\varphi=(d+\delta) \Psi$, with $\Psi \in \Omega^{*}(M)$,

$$
\begin{aligned}
0 & =((d+\delta) u, \varphi)=(u,(d+\delta) \varphi)=(u,(d \delta+\delta d) \Psi) \\
& =(d u, d \Psi)+(\delta u, \delta \Psi) .
\end{aligned}
$$

Let $\left\{\Psi_{n}\right\}_{n}$ be a sequence in $\Omega^{*}(M)$ converging to $u$ in the \|\|$_{1}$-norm. Then we have from (6.1)

$$
0=\lim _{n \rightarrow \infty}\left[\left(d u, d \Psi_{n}\right)+\left(\delta u, \delta \Psi_{n}\right)\right]=(d u, d u)+(\delta u, \delta u) .
$$

Hence $d u=0=\delta u$.

In this section we use some constructions and results of Atiyah-Singer [A.S., §6].

Let

$$
\bar{H}_{s}^{p}=H_{s}^{p}(M) \otimes_{\mathrm{R}} \mathrm{C}, \quad \overline{\mathscr{T}}_{s}^{p}=\mathcal{F}_{s}^{p}(M) \otimes_{\mathrm{R}} \mathrm{C}
$$


for any $0<p<\operatorname{dim} M,-\infty<s<+\infty$, and

$$
\bar{H}_{s}^{*}=\bigoplus_{p} \bar{H}_{s}^{p}(M), \quad \mathscr{H}_{s}^{*}=\bigoplus_{p} \mathcal{H}_{s}^{p} .
$$

Suppose $M$ is even dimensional, $\operatorname{dim} M=2 l$.

Let $\tau_{p}: \bar{H}_{s}^{p} \rightarrow \bar{H}_{s}^{m-p}, m=\operatorname{dim} M$, be defined such that

$$
\tau_{p}(\alpha)=i^{p(p-1)+l} * \alpha,
$$

and

$$
\tau=\bigoplus_{p} \tau_{p}: \bar{H}_{s}^{*} \rightarrow \bar{H}_{s}^{*}
$$

It is easy to check that $\tau^{2}=1$.

Let $\bar{H}_{s}^{ \pm}$denote the eigenspaces of $\tau$ corresponding to the eigenvalues \pm 1 . As in the smooth case, we can prove that

$$
D_{\tau}=-\tau D \text {. }
$$

From now on let $D^{ \pm}$denote the restrictions of $D$ to $H_{1}^{ \pm}$. From (6.6) we deduce that

$$
D \pm\left(\bar{H}_{1}^{ \pm}\right) \subseteq{\overline{H_{0}}}^{ \pm} \text {. }
$$

The operators $D^{+}, D^{-}$are adjoint to one another, i.e. for any $\omega \in \bar{H}_{1}^{+}$, $\theta \in \bar{H}_{1}^{-}$, we have

$$
\left(D^{+} \omega, \theta\right)=\left(\omega, D^{-} \theta\right)
$$

where (, ) denotes the canonical hermitian scalar product which arises from the scalar product in $H_{0}^{*}(M)$.

Let $\overline{\mathscr{K}}_{1}^{ \pm}=\overline{\mathscr{K}}_{1}^{*} \cap \bar{H}_{1}^{ \pm}$. It is clear that $\overline{\mathscr{F}}_{s}^{*}$ is invariant under $\tau$; hence $\overline{\mathscr{K}}_{1}^{*}$ decomposes as

$$
\overline{\mathscr{K}}_{1}^{*}=\overline{\mathscr{T}}_{1}^{+} \oplus \overline{\mathscr{K}}_{1}^{-}
$$

where $\overline{\mathscr{T}}_{1}^{ \pm}$are the eigenspaces of $\tau$ in $\overline{\mathscr{T}}_{1}^{*}$.

It is known that any compact oriented PL-manifold is a Poincaré complex, that is

$$
(\alpha, \beta) \mapsto \int_{M} \alpha \wedge \beta, \quad \alpha \in H^{p}(M, \mathbf{R}), \quad \beta \in H^{q}(M, \mathbf{R}),
$$

is a nondegenerate pairing.

In particular, if $\operatorname{dim} M=2 l=4 \lambda$, then

$$
\alpha, \beta \mapsto\langle\alpha, \beta\rangle=\int \alpha \wedge \beta, \quad \alpha, \beta \in H^{2 \lambda}(M, \mathbf{R}),
$$

is a nondegenearte bilinear form and it will be called the Poincare scalar product.

The signature of the bilinear form (6.10) is by definition the signature of $M$, written Sig $M$. 
Let us define

$$
\begin{aligned}
& R=\chi_{1}^{2 \lambda} \mathcal{G}_{1}^{2 \lambda} \subseteq H^{2 \lambda}(M, \mathbf{R}), \\
& \bar{R}=\chi_{1}^{2 \lambda} \overline{\mathcal{G}}_{1}^{2 \lambda} \subseteq H^{2 \lambda}(M, \mathrm{C}),
\end{aligned}
$$

and let $S$ denote the orthogonal complement of $R$ in $H^{2 \lambda}(M, R)$ with respect to the Poincare scalar product.

Proposition 6.2. If $M$ is a compact, oriented, PL-manifold without boundary, with flat Riemannian metric, $\operatorname{dim} M=4 \mu$, and $R, S$ are defined as above then

(i) $H^{2 \mu}(M, \mathbf{R})=R \oplus S$,

(ii) the restriction of the Poincaré scalar product to $R, S$ is still nondegenerate.

Proof. (i) Let $R^{ \pm}=\chi_{1}^{2 \mu} \mathcal{Y C}_{1}^{ \pm}$. From (6.9) we deduce that $R=R^{+} \oplus R^{-}$. Let $h_{1}, h_{2} \in R^{+}$; then

$$
\left\langle h_{1}, h_{2}\right\rangle=\left(h_{1}, * h_{2}\right)=\left(h_{1}, h_{2}\right)
$$

Let $h_{1}, h_{2} \in R^{-}$; then

$$
\left\langle h_{1}, h_{2}\right\rangle=\left(h_{1}, * h_{2}\right)=\left(h_{1},-h_{2}\right)=-\left(h_{1}, h_{2}\right) \text {. }
$$

Let $h_{1} \in R^{+}, h_{2} \in R^{-}$; then

$$
\begin{aligned}
\left(h_{2}, h_{1}\right) & =\left(h_{2}, * h_{1}\right)=\left\langle h_{2}, h_{1}\right\rangle=\left\langle h_{1}, h_{2}\right\rangle \\
& =\left(h_{1}, * h_{2}\right)=\left(h_{1},-h_{2}\right)=-\left(h_{1}, h_{2}\right) ;
\end{aligned}
$$

hence $\left\langle h_{1}, h_{2}\right\rangle=0$.

(6.12)-(6.14) show that the Poincare scalar product restricted to $R$ is still nondegenerate.

(ii) Let $e_{1}, \ldots, e_{r}$ be an orthonormal base in $R^{+}$(with respect to $\langle$,$\rangle ) and$ let $e_{r+1}, \ldots, e_{r+s}$ be an orthonormal base in $R^{-}$. Let $e_{r+s+1}, \ldots, e_{r+s+t}$ be such that $e_{1}, \ldots, e_{r}, e_{r+1}, \ldots, e_{r+s}, e_{r+s+1}, \ldots, e_{r+s+t}$ is a base in $H^{2 \mu}(M, \mathbf{R})$.

Let $f=\Sigma_{i} x^{i} e_{i}, x^{i} \in \mathbf{R}$, be any element in $S$. Then, if $\left\langle e_{i}, e_{j}\right\rangle=a_{i j}$, $1<i<r+s, r+s+1<j<r+s+t$, we have

$$
\begin{aligned}
& 0=\left\langle e_{i}, f\right\rangle=x^{i}+\sum_{r+s+1<j<r+s+i} a_{i j} x^{j}, \quad 1<i<r, \\
& 0=\left\langle e_{i}, f\right\rangle=-x^{k}+\sum_{r+s+1<j<r+s+i} a_{k j} x^{j}, \quad r+1<k<s .
\end{aligned}
$$

From these equations we deduce that $\operatorname{dim} S=t$, because we can take $x^{r+s+1}, \ldots, x^{r+s+t}$ arbitrary, while $x^{1}, \ldots, x^{r+s}$ are given by (6.15).

We can choose $e^{r+s+1}, \ldots, e^{r+s+t}$ in $S$. The Poincare scalar product in this new base is given by a matrix of the form 


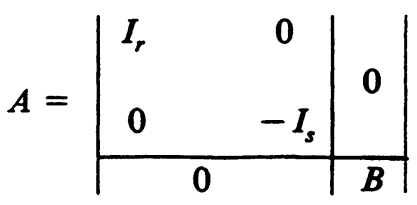

As the Poincare scalar product is nondegenerate, it is $\operatorname{det} A \neq 0$, and therefore det $B \neq 0$, which shows that the Poincaré scalar product, restricted to $S$, is nondegenerate (its matrix in the base $e^{r+s+1}, \ldots, e^{r+s+t}$ is $B$ ).

Let us define

$$
\text { Index } D=\operatorname{dim} \operatorname{Ker} D^{+}-\operatorname{dim} \operatorname{Ker} D^{-} \text {. }
$$

THEOREM 6.3. Let $M$ be any compact, oriented, PL-manifold with Riemannian metric, $\partial M=\phi$, of dimension $4 \mu$. Then

$$
\text { Index } D=\operatorname{Sig} M+\theta
$$

where

$$
\theta=-\operatorname{Sig}(S,\langle,\rangle) \text {. }
$$

ProOf. The proof of the fact that Index $D$ depends only on the Hodge homomorphism in the middle dimension proceeds as in the smooth case (see Atiyah-Singer [A.S., \$6]).

From Theorem 5.2(i), we have

$$
\mathcal{K}_{1}^{2 \mu_{1}+} \simeq R^{+}, \quad \mathcal{F}_{1}^{2 \mu_{,}-} \simeq R^{-} .
$$

From Proposition 6.1 and repeating the specific argument from [A.S., \$6], we obtain

$$
\text { Index } D=\operatorname{dim} \mathcal{K}_{1}^{2 \mu_{1}+}-\operatorname{dim} \mathcal{K}_{1}^{2 \mu_{3}-} .
$$

From (6.17), (6.18) we deduce

$$
\text { Index } D=\operatorname{dim} R^{+}-\operatorname{dim} R^{-} .
$$

But, from Proposition 6.2 we obtain

$$
\begin{aligned}
\operatorname{Sig} M & =\operatorname{Sig}(R,\langle,\rangle)+\operatorname{Sig}(S,\langle,\rangle) \\
& =\left(\operatorname{dim} R^{+}-\operatorname{dim} R^{-}\right)+\operatorname{Sig}(S,\langle,\rangle) .
\end{aligned}
$$

Now (6.16) is a direct consequence of (6.19) and (6.20).

Appendix I. Partition of unity. As before, $M^{m}$ denotes a combinatorial manifold with a flat metric $\Gamma$.

Let $f$ be a $C^{\infty}$ real function on $M$. Let $x$ be any point in $M$; let $\tau \in M$ denote the unique simplex of $M$ which contains $x$ as an interior point.

We say that $f$ has "property $F^{\prime}$ in $x$ iff, for every $m$-simplex $\sigma^{m}, \tau \in \sigma^{m} \in$ $M$, there exists a small neighborhood $U$ of $x, U \subset \sigma^{m}$, such that for any segment $l \subset \sigma^{m}, x \in l$, which is normal to $\tau$, the function $f \mid(l \cap U)$ is constant. 
We define

$$
F=\left\{f \mid f \in C^{\infty}(M, \Gamma), f \text { has property } F \text { in any point } x \in M\right\} .
$$

It is clear that for any two disjoint closed sets $A_{1}, A_{2}$ in $M$, there exists an $f \in F$ which separates them. Therefore, for any locally finite open covering of $M$ there exists a subordinate partition of unity with function in $F$.

Appendix II. Proof of Lemma 4.6 (ChaPTER 3, \$4). We need the following:

Proposition II.1. Let $\tilde{U}$ be a closed star neighborhood in the combinatorial manifold $M^{m-1}$ of dimension $\boldsymbol{m}-1$.

Let $x_{\sigma}(\sigma$ any $(m-1)$-simplex in $\tilde{U})$ denote fixed affine coordinates as in II. 1 , and let $t$ denote the standard coordinate on $\mathbf{R}$.

Then for any $\omega \in \Omega^{*}(\tilde{U} \times[-1,1])$, there exists a sequence of forms

$$
\omega^{i} \in \Omega^{*}(\tilde{U}) \otimes_{\mathrm{R}} \Omega^{*}([-1,1]), \quad 1<i<\infty,
$$

such that for any maximal simplex $\sigma \in \tilde{U}$, and for any partial derivative $D_{x, t}^{\alpha}$,

$$
\lim _{i \rightarrow \infty} \sup _{\sigma \times[-1,1]} D_{x, i}^{\alpha}\left(\omega_{\sigma, I}^{i}-\omega_{\sigma, I}\right)=0
$$

here I indexes components of forms.

Proof of Proposition II.1. The argument used by de $\mathbf{R h a m}[\mathbf{R}$, Theorem 2] for proving the same result in the case of smooth manifolds can be used, although in our case a technical difficulty appears: partial derivatives with respect to the $x_{\sigma}$ 's do not lead to smooth forms on $U \times[1,1]$. Fortunately, $\partial^{r} \omega / \partial t^{r}, 0<r<\infty$, is a $C^{\infty}$-form on $\tilde{U} \times[-1,1]$.

We may assume that Supp $\omega \subset \tilde{U} \times(-1,1)$.

Let $\varepsilon>0$ be any positive number and let $\rho$ be any positive integer. There exists a $\delta>0$ such that for any $t^{\prime}, t^{\prime \prime} \in(-1,1),\left|t^{\prime}-t^{\prime \prime}\right|<\delta$,

$$
\left|\frac{\partial^{m p}}{\left(\partial x^{1}\right)^{p} \ldots\left(\partial x^{m-1}\right)^{p} \partial t^{p}} \omega_{\sigma, I}^{i}\left(x, t^{\prime}\right)-\frac{\partial^{m p}}{\left(\partial x^{1}\right)^{p} \ldots\left(\partial x^{m-1}\right)^{p} \partial t^{p}} \omega_{\sigma, I}^{i}\left(x, t^{\prime \prime}\right)\right|<\varepsilon .
$$

Let $N$ be such that $1 / N<\delta / 3$. Let $\left\{\varphi_{j}\right\}, 1<j<N$, be a partition of unity on $[-1,1]$ such that diam Supp $\varphi_{j}<\frac{2}{3} \delta$, and let choose $t_{j} \in \operatorname{Supp} \varphi_{j}$.

Then we define the form

$$
P(x, t)=\sum_{j=1}^{N} \frac{\partial^{p}}{\partial t^{p}} \omega\left(x, t_{j}\right) \varphi_{j}(t),
$$

and let $Q$ denote the $p$ th order primitive of $P$, i.e.,

$$
\begin{aligned}
\frac{\partial^{r}}{\partial t^{r}} Q(x,-1) & =0, \quad 0<r<p-1, \\
\frac{\partial^{p}}{\partial t^{p}} Q(x, t) & =P(x, t) .
\end{aligned}
$$


From now on, the proof of Proposition II.1 goes on as in de Rham [R, p. 8], with the same notations.

The proof of Lemma 4.6 follows from Proposition II.1 as in the case of smooth manifolds. For its proof see Schwartz [Sc].

\section{REFERENCES}

[A] S. Agmon, Lectures on elliptic value problem, Van Nostrand, Princeton, N. J., 1965.

[A.S.] M. F. Atiyah and I. M. Singer, The index of elliptic operators. III, Ann. of Math. (2) 87 (1968), 546-604.

[B.R.] P. Bidal and G. de Rham, Les formes différentielles harmoniques, Comment. Math. Helv. 19 (1946), $1-49$.

[F] A. Friedman, Partial differential equations, Holt, Rinehart and Winston, San Francisco, California, 1969.

[Hd] W. V. D. Hodge, The theory and applications of harmonic integrals, 2nd ed., Cambridge Univ. Press, Cambridge, 1952.

[H] L. Hörmander, An introduction to complex analysis in several variables, Van Nostrand, Princeton, N. J., 1966.

[M] J. Munkres, Obstruction to impasing differentiable structures, Illinois J. Math. 8 (1964), 361-375.

[Mi] J. Milnor, Microbundles. I, Topology 3 (Suppl. 1) (1964), 53-84.

[P] R. Palais, Seminar on the Atiyah-Singer index theorem, Ann. of Math. Studies, no. 57, Princeton Univ. Press, Princeton, N. J., 1965.

[R] G. de Rham, Variétés différentiables, formes, currants, formes harmonique, Actualités Sci. Indus., no. 1222, Hermann, Paris, 1960.

[S] I. M. Singer, Future extensions of index theory and elliptic operators, Ann of Math. Studies, no. 70, Princeton Univ. Press, Princeton, N. J., 1971.

[Sc] L. Schwartz, Théorie des distributions, Hermann, Paris, 1966.

[Su ] D. Sullivan, Differential forms and the topology of manifolds, Proc. Tokyo Conf. on Manifolds, Univ. of Tokyo Press, Tokyo, 1973.

[Su ] , Geometric topology. I, M.I.T. Notes, 1969.

[T] N. Tanaka, A differential geometric study on strongly pseudoconoex manifolds, Kinokuniya Book-Store Co., Tokyo, 1975.

Department of mathematics, Massachusetts institute of Tbchinology, Cambridge, MASSACHUSETTS 02139

Department of MATHEMATICS, Lecce UntVerstTy, Lecce, ItALY

Current address: Department of Mathematics, California Institute of Technology, Pasadena, California 91125 